Pacific Journal of Mathematics

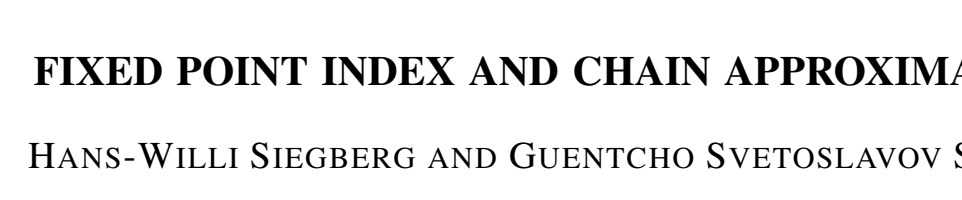




\title{
FIXED POINT INDEX AND CHAIN APPROXIMATIONS
}

\author{
H. W. Siegberg and G. Skordev
}

Localizing the Lefschetz number of certain chain approximations of upper semi continuous multivalued mappings a new approach to fixed point index is given. It turns out that this fixed point index satisfies the commutativity property as well as the mod- $p$ property (known from the singlevalued case). In particular, in the single-valued case the proof of the mod- $p$ property is a natural consequence of a corresponding property of (global) Lefschetz number.

Introduction. The aim of the present note is to provide a unified approach to fixed point index theory for single-valued mappings as well as for certain classes of multivalued mappings (e.g., acyclic mappings) on compact polyhedra.

Fixed point index theory was initiated in $1926 / 1927$ by S. Lefschetz [27] with his celebrated fixed point formula on manifolds (with triangulation), and it was generalized one year later by H. Hopf [23] to compact polyhedra. (For a good survey of fixed point index theory we recommend [14].) Later, after the classical paper of J. Leray and J. Schauder [30] (1934) the problem of generalizing and extending the Leray-Schauder theory resp. the fixed point index theory arose in a natural way. In the forties J. Leray [28], [29] succeeded in developing an index theory for the category of 'convexoid spaces' which are in some sense homological generalizations of simplicial complexes, see also [4]. The techniques introduced by Leray are very general; indeed, his cohomology theory is the precursor of modern sheaf cohomology.

In 1953 B. O'Neill [34] rederived-for the category of compact polyhedra-the principle results of Leray's theory (and Hopf's results), and, moreover, he proved that fixed point index is uniquely determined by certain axioms (in the category of compact polyhedra). In 1960 F. E. Browder [5] extended fixed point index theory to 'semi-complexes' by finding appropriate algebraic analogues of the properties of fixed point index for chain mappings.

All these results use definitions of fixed point index based on certain induced chain mappings. A purely homological approach to fixed point index (on manifolds) was discovered in 1965 by A. Dold [9], [10], see also [11] where Dold extends his technique to coincidence problems.

There are essentially two ways to handle multivalued fixed point problems. The first one is based on homological arguments and roots 
in the early work of L. Vietoris [44] (and of E. Begle [1]) where the 'Vietoris-Begle mapping theorem' is proved, and where for acyclic maps induced homomorphisms in (Vietoris-) homology are defined. Using the Vietoris (-Begle) mapping theorem S. Eilenberg and D. Montgomery [12] in 1946 generalized the Lefschetz fixed point theorem to acyclic mappings (on compact metric ANR's). The other way to deal with multivalued fixed point problems is influenced by homotopic considerations, and detects fixed points of the multivalued map $\Phi$ by fixed points of single-valued 'approximations' of $\Phi$. Early examples of this procedure were given by J. von Neumann [33] and by S. Kakutani [24]. For a survey of both methods we recommend [2].

The first approach to degree resp. fixed point index of multivalued (convex or acyclic) mappings goes back to A. Granas [19] and to A. Granas and J. W. Jaworowski [20] in 1959. Further approaches to degree of multivalued mappings based on single-valued approximations were introduced by A. Cellina and A. Lasota [8] and by Yu. G. Borisovič et al. [3].

In $1970 \mathrm{~B}$. D. Calvert [7] gave a homological definition of fixed point index for acyclic maps which runs along lines similar to [9]: Using the Vietoris-Begle mapping theorem Calvert applies Dold's technique to the induced homomorphism of the acyclic map. In [21] and [26] there are similar homological approaches. We refer also to [15] where Calvert's index of 'repulsive' fixed points is computed.

A common lack of all these approaches to fixed point index of multivalued (e.g., acyclic) maps is that it is unclear whether the commutativity property holds, see [7], [15], [18]. Beside some special cases there is no proof for this property [7], [15]. Moreover, it is not known whether the mod-p property in the sense of ZabrerkoKrasnosel'skiY-Steinlein, $i(X, f, U) \equiv i\left(X, f^{p}, U\right) \bmod p, p$ prime, is satisfied, see [15], [18], [37].

In this paper we will give an affirmative answer to these two questions provided an alternative definition of fixed point index is used. We shall define a fixed point index for a multivalued (acyclic) mapping by associating to this map a chain map ("chain approximation"), and by computing the 'local' Lefschetz number (in the sense of O'Neill and Browder) of that chain map.

The paper is organized as follows:

In Chapter I we describe algebraic analogues of properties of the fixed point index for chain mappings (including commutativity and the mod-p property). In Chapter II chain approximations and corresponding fixed point indices are discussed, and in Chapters III and IV the results of I and II are applied to acyclic mappings on polyhedra. Finally, we remark that in the single-valued case proofs 
(e.g., the proof of the mod-p property) become considerably easier provided classical simplicial approximation is used.

Acknowledgment. We would like to thank Prof. H. O. Peitgen for valuable discussions and for helpful suggestions.

I. Index and chain mappings. Since the fundamental papers of Leray [28], O'Neill [34], and, in particular, of Browder [5] one knows that 'local Lefschetz numbers' of chain mappings have algebraic properties which are in analogy to corresponding properties of the fixed point index (Homotopy invariance, Normalization, Additivity, Commutativity). In the following we will recapitulate this fact, and, moreover, we will find a mod- $p$ property which is easy to prove, and which provides the corresponding mod-p property for the fixed point index.

In what follows $K=(K, \tau)$ and $L=(L, \mu)$ will always denote compact polyhedra with fixed triangulations $\tau$ resp. $\mu$.

o. Preliminaries and notations. For $l \in N^{1}$ and a triangulation $\tau$ we denote by $\tau^{l}$ the lth barycentric subdivision of $\tau$. A subset $U \subset K$ is called polyhedral provided there is an integer $l \in N$ such that $\tau^{l}$ induces a triangulation of the closure $\bar{U}$ of $U$. Let $B \subset K$ and $k \in N$. The kth star of $B$ in $\tau$,

$$
s t^{k}(B, \tau) \text {, }
$$

is defined recursively as follows:

$$
\begin{aligned}
& s t^{1}(B, \tau):=s t(B, \tau):=\cup\{\sigma \in \tau \mid \sigma \cap B \neq \varnothing\} \\
& s t^{k}(B, \tau):=s t\left(s t^{k-1}(B, \tau), \tau\right), \quad k>1 .
\end{aligned}
$$

(Simplices are always assumed to be closed.)

For $l \in N$ and a field $\boldsymbol{F}$ denote by $C_{*}(K, l)$ the oriented chain complex $C_{*}\left(K, \tau^{l} ; \boldsymbol{F}\right)$, see $[39$, p. 159].

If $C$ and $C^{\prime}$ are two chain complexes (over $\boldsymbol{F}$ ) denote by hom $\left(C, C^{\prime}\right)$ the space of all chain mappings $\varphi: C \rightarrow C^{\prime}$. Let $c \in$ $C_{*}(K, \tau)$. The carrier of $c, \operatorname{carr}(c)$, is the smallest polyhedral subset $X \subset K$ such that $c \in C_{*}(X, \tau)$, see $[10$, p. 198].

Definition. Let $\varphi: C_{*}(K, \tau) \rightarrow C_{*}(K, \tau)$ be a chain mapping, and let $\sigma, \sigma^{\prime} \in C_{n}(K, \tau)$ be two simplices of dimension $n$.

$\sigma^{\prime}$ is contained in $\boldsymbol{\phi}(\boldsymbol{\sigma}), \sigma^{\prime} \in \boldsymbol{\phi}(\boldsymbol{\sigma})$, provided $\sigma^{\prime}$ is contained in the irreducible representation of $\varphi(\sigma)$ (with respect to the basis of $C_{n}(K, \tau)$ which consists of all simplices of dimension $n$ ) with a nonzero coefficient.

$1 N=$ natural numbers. 
$\sigma$ is called a fixed simplex of $\phi$ iff $\sigma \in \varphi(\sigma)$.

Let $l \in N$. Then $b: C_{*}(K, \tau) \rightarrow C_{*}(K, l)$ is the subdivision chain map which maps each chain of $C_{*}(K, \tau)$ into its $l$ th barycentric subdivision [10, p. 40]. By $\chi: C_{*}(K, l) \rightarrow C_{*}(K, \tau)$ we denote any chain mapping which is induced by a simplicial approximation of Id: $\left(K, \tau^{l}\right) \rightarrow(K, \tau)$, [10, p. 119].

Let $V=\left\{V_{q}\right\}_{q \in N}$ be a graded $\boldsymbol{F}$-vector space with $\operatorname{dim} V<\infty$, and let $\beta=\left\{\beta_{q}\right\}_{q \in N}$ be an endomorphism of $V$ of degree zero.

Then

$$
\Lambda(\beta):=\sum_{q \in N}(-1)^{q} \operatorname{tr}\left(\beta_{q}\right) \quad(\operatorname{tr}=\text { trace })
$$

is called the Lefschetz number of $\beta,[10$, p. 208].

\section{Index systems.}

Definition 1.1. Let $U \subset K$ be open and polyhedral. A graded set

$$
M(K, U)=\left\{M(K, U)_{k}\right\}_{k \in N}
$$

where $M(K, U)_{k} \subset \operatorname{hom}\left(C_{*}(\bar{U}, k), C_{*}(K, k)\right)$, is called Index system (I-system) on $\boldsymbol{U}$ provided the following properties are satisfied:

There is an integer $k_{0}=k_{0}(M(K, U))$ such that for all $l, k \in N$, $l \geqq k \geqq k_{0}$, and for all $\varphi_{1} \in M(K, U)_{k}, \varphi_{2} \in M(K, U)_{l}$

(i ) $\varphi_{1}$ and $\chi \varphi_{2} b$ are admissible homotopic, i.e., the diagram

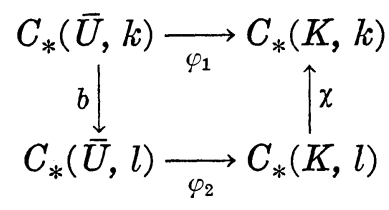

is homotopy commutative with a chain homotopy $D$ which satisfies the following boundary condition, for any simplex $\sigma \in \tau^{k}$ with $\operatorname{st}(\sigma, k) \cap \partial U \neq \varnothing$,

$$
\operatorname{carr} D \sigma \cap s t(\sigma, k)=\varnothing \text {. }
$$

$\left(s t\left(\cdot, \tau^{k}\right)\right.$ is abbreviated by $s t(\cdot, k)$ in the following.)

(ii) $\left.\varphi_{2} b \chi\right|_{\bar{U}}$ and $\varphi_{2}$ are admissible homotopic.

$$
\left(\left.\chi\right|_{\bar{U}}:=\left.\chi\right|_{C_{*}(\bar{U}, l)}\right)
$$

REMARK. In the definition above we did assume that $U$ is already polyhedral in $\tau$, otherwise we have a graded set $\left\{M(K, U)_{k}\right\}_{k \geqq l_{0}}$ where $U$ is polyhedral in $\tau^{l_{0}}$

To any $I$-system $M(K, U)$ we associate a 'local' Lefschetz number which will be called the index of $M(K, U)$. 
Lemma AND Definition 1.2. Let $M(K, U)$ be an I-system, and let $l \geqq k \geqq k_{0}(M(K, U))$.

Let $\varphi_{1} \in M(K, U)_{k}, \varphi_{2} \in M(K, U)_{l}$.

Then

$$
\Lambda\left(\pi \varphi_{1}\right)=\Lambda\left(\pi \varphi_{2}\right),
$$

where $\pi=\pi_{\bar{U}}: C_{*}(K, k) \rightarrow C_{*}(U, k)$ resp. $C_{*}(K, l) \rightarrow C_{*}(\bar{U}, l)$ are the natural projections.

$$
I(M(K, U)):=\Lambda(\pi \varphi) \in \boldsymbol{F}, \quad \varphi \in M(K, U)_{k}, \quad k \geqq k_{0}(M(K, U)),
$$

is called the index of the I-system $(M(K, U)$.

Proof. Since $\varphi_{1}$ and $\chi \varphi_{2} b$ are admissible homotopic we have $\Lambda\left(\pi \varphi_{1}\right)=\Lambda\left(\pi \chi \varphi_{2} b\right),\left[5\right.$, p. 274]. Moreover, one computes $\Lambda\left(\pi \chi \varphi_{2} b\right)=$ $\Lambda\left(\chi_{\pi} \varphi_{2} b\right)$. Since the trace is commutative, and since $\varphi_{2} b \chi_{\mid \bar{U}}$ and $\varphi_{2}$ are admissible homotopic, Browder's lemma applies again,

$$
\Lambda\left(\chi_{\pi \varphi_{2}} b\right)=\Lambda\left(\pi \varphi_{2}\right) \text {. }
$$

Hence, $\Lambda\left(\pi \varphi_{1}\right)=\Lambda\left(\pi \varphi_{2}\right)$.

In the following we list some properties of $I(M(K, U))$ :

Proposition 1.3 (Additivity). Let $M=M(K, U)$ be an I-system, and let $U_{1}, U_{2} \subset U$ be open, disjoint subsets which are polyhedral. Assume that

$$
M_{i}:=\left.M(K, U)\right|_{\bar{U}_{i}}:=\left\{\left.M(K, U)_{k}\right|_{\bar{U}_{i}}\right\}_{k \in N},
$$

where $\left.M(K, U)_{k}\right|_{\bar{U}_{i}}:=\left\{\left.\varphi\right|_{\bar{U}_{i}} \mid \varphi \in M(K, U)_{k}\right\}$, are I-systems for $i=1,2$. Assume further that the following condition is satisfied:

There is an integer $k^{\prime} \in N$ such that for all $k \geqq k^{\prime}$ there is a $\operatorname{map} \varphi \in M(K, U)_{k}$ with the following property, for any simplex $\sigma \in \tau^{k}$ with $\operatorname{st}(\sigma, k) \cap\left(\bar{U} \backslash\left(U_{1} \cup U_{2}\right)\right) \neq \varnothing$,

$$
\operatorname{carr} \varphi(\sigma) \cap \operatorname{st}(\sigma, k)=\varnothing \text {. }
$$

Then

$$
I(M)=I\left(M_{1}\right)+I\left(M_{2}\right) .
$$

Definition 1.4. Let $M(K, U)$ and $M^{\prime}(K, U)$ be $I$-systems. $M(K, U)$ and $M^{\prime}(K, U)$ are called homotopic, provided there is an integer $k^{\prime \prime} \in N$ such that for all $k \geqq k^{\prime \prime}$ there are maps $\varphi \in M(K, U)_{k}$ and $\varphi^{\prime} \in M^{\prime}(K, U)_{k}$ which are admissible homotopic.

Proposition 1.5 (Homotopy Invariance). Let $M(K, U)$ and $M^{\prime}(K, U)$ be two I-systems which are homotopic. Then 


$$
I(M(K, U))=I\left(M^{\prime}(K, U)\right)
$$

2. Commutativity and mod-p property. If $C, C^{\prime}, C^{\prime \prime}$ are chain complexes (over $\boldsymbol{F}$ ), and if $A \subset \operatorname{hom}\left(C, C^{\prime}\right), B \subset \operatorname{hom}\left(C^{\prime}, C^{\prime \prime}\right)$, define

$$
B \circ A:=\{\psi \circ \varphi \mid \psi \in B, \varphi \in A\} \subset \operatorname{hom}\left(C, C^{\prime \prime}\right) .
$$

Proposition 1.6 (Commutativity). Let $U \subset K, V \subset L$ be open and polyhedral subsets of $K$ resp. $L$, and let

$$
A(K, L)=\left\{A(K, L)_{k}\right\}_{k \in N}, \quad A(L, K)=\left\{A(L, K)_{k}\right\}_{k \in N}
$$

be graded sets where

$$
\begin{aligned}
& A(K, L)_{k} \subset \operatorname{hom}\left(C_{*}(K, k), C_{*}(L, k)\right) \\
& A(L, K)_{k} \subset \operatorname{hom}\left(C_{*}(L, k), C_{*}(K, k)\right) .
\end{aligned}
$$

Assume

$$
A(K, L) \circ A(L, K):=\left\{A(K, L)_{k} \circ A(L, K)_{k}\right\}_{k \in N}
$$

and

$$
A(L, K) \circ A(K, L):=\left\{A(L, K)_{k} \circ A(K, L)_{k}\right\}_{k \in N}
$$

are $I$-systems on $U$ resp. $V$, and denote them by $M(K, U)$ resp. $M(L, V)$.

Assume further that the following condition is satisfied, there is an integer $\tilde{k} \in N$ such that for all $k \geqq \tilde{k}$ |there are maps $\varphi \in A(K, L)_{k}, \psi \in A(L, K)_{k}$ with the following properties:

(i) For any simplex $\sigma \in \mu^{k}, \sigma \subset \bar{V}$,

$$
\sigma_{1} \in \psi(\sigma), \sigma_{1} \not \subset \bar{U} \Longrightarrow \sigma \notin \varphi\left(\sigma_{1}\right)
$$

(ii) For any simplex $\sigma \in \tau^{k}, \sigma \subset \bar{U}$,

$$
\sigma_{1} \in \varphi(\sigma), \sigma_{1} \not \subset \bar{V} \Longrightarrow \sigma \notin \psi\left(\sigma_{1}\right) \text {. }
$$

Then

$$
I(M(K, U))=I(M(L, V))
$$

Proof. Choose $k$ sufficiently large such that

$$
I(M(K, U))=\Lambda\left(\pi_{\bar{U}} \circ \psi \circ \bar{\varphi}\right), \quad I(M(L, V))=\Lambda\left(\pi_{\bar{V}} \circ \varphi \circ \bar{\psi}\right),
$$

where $\varphi \in A(K, L)_{k}, \psi \in A(L, K)_{k}, \bar{\varphi}:=\left.\varphi\right|_{\bar{U}}, \bar{\psi}:=\left.\psi\right|_{\bar{v}}$, and where $\varphi$ and $\psi$ satisfy properties (i) and (ii). Choose bases of $C_{i}(K, k)$ and $C_{i}(L, k)$ consisting of simplices of dimension $i, 0 \leqq i \leqq \operatorname{dim} K, \operatorname{dim} L$, and compute the matrix representations of

$$
\begin{aligned}
& \pi_{\bar{U}^{\circ}} \psi \circ \bar{\varphi}: C_{i}(\bar{U}, k) \longrightarrow C_{i}(\bar{U}, k) \\
& \pi_{\bar{V}} \circ \varphi \circ \bar{\psi}: C_{i}(\bar{V}, k) \longrightarrow C_{i}(\bar{V}, k)
\end{aligned}
$$


with respect to those bases.

It follows immediately that the traces of $\pi_{\bar{U}} \circ \psi \circ \bar{\varphi}$ and $\pi_{\bar{v}} \circ \varphi \circ \bar{\psi}$ coincide, and, hence, we obtain

$$
I(M(K, U))=I(M(L, V))
$$

Proposition 1.7 (Mod-p Property). Let $\boldsymbol{F}=Z_{p}, p$ prime. Let $U \subset K$ be open and polyhedral.

Let $A(K, K)=\left\{A(K, K)_{k}\right\}_{k \in N}$ be a graded set where

$$
A(K, K)_{k} \subset \operatorname{hom}\left(C_{*}(K, k), C_{*}(K, k)\right) .
$$

Assume that $A(K, K)$ and $A(K, K) \circ A(K, K) \circ \cdots \circ A(K, K)(p$ times $)$ are $I$-systems on $U$, and denote them by $M(K, U)$ and $M^{p}(K, U)$. Assume further that the following condition is satisfied, there is an integer $\bar{k} \in N$ such that for all $k \geqq \bar{k}$ there is a map $\varphi \in A(K, K)_{k}$ with the following property:

For any simplex $\sigma \in \tau^{k}, \sigma \subset \bar{U}$, with $\sigma \in \varphi^{p}(\sigma)$,

$$
\sigma_{1} \in \varphi^{p-j}(\sigma), \sigma_{1} \not \subset \bar{U}, \sigma_{1} \in \tau^{k} \Longrightarrow \sigma \in \varphi^{j}\left(\sigma_{1}\right) \text { for all } j, 1 \leqq j<p .
$$

Then

$$
I(M(K, U))=I\left(M^{p}(K, U)\right)\left(\text { in } Z_{p}\right)
$$

Proof. We give a proof for $p=2$, for $p>2$ the proof runs along the same lines.

Choose $k$ sufficiently large such that

$$
I(M(K, U))=\Lambda(\pi \bar{\varphi}), \quad I\left(M^{2}(K, U)\right)=\Lambda(\pi \varphi \bar{\varphi}),
$$

where $\varphi \in A(K, K)_{k}, \bar{\varphi}=\left.\varphi\right|_{\bar{U}}$, and where $\varphi$ satisfies the assumption of the proposition. Using the lemma in [36, p. 441] ("Fermat's theorem") we have

$$
\Lambda(\pi \bar{\varphi} \pi \bar{\varphi})=\Lambda(\pi \bar{\varphi}) \quad\left(\text { in } Z_{2}\right) .
$$

But the assumption of the proposition implies that

$$
\left.\operatorname{tr} \pi \bar{\varphi} \pi \bar{\varphi}\right|_{C_{i}(\bar{U}, k)}=\left.\operatorname{tr} \pi \varphi \bar{\varphi}\right|_{C_{i}(\bar{U}, k)}
$$

for $i, 0 \leqq i \leqq \operatorname{dim} K$. Hence the mod-p property follows.

II. Approximation systems and fixed point indices. In this chapter we will study chain mappings and their approximation properties. It turns out that in order to define a fixed point index à la O'Neill [34] or Browder [5] one does not need a chain mapping which is induced by simplicial approximation but a chain map which has 
certain approximation properties. We will show how such "approximation systems" lead to fixed point index theories even for multivalued mappings in a natural way. In the following $(K, \tau),(L, \mu)$ and $(M, \nu)$ will always denote compact polyhedra with fixed triangulations $\tau, \mu$ and $\nu$.

Let $\Phi: K \rightarrow K$ be an upper semi continuous (u.s.c.) multivalued map $^{1}$ i.e., the graph $\{(x, y) \in K \times K \mid y \in \Phi(x)\}$ is closed, and let $U \subset$ $K$ be an open subset of $K$. We will say that the triple $(K, \Phi, U)$ is admissible iff $x \notin \Phi(x)$ for all $x \in \partial U$.

\section{Approximation systems.}

Definition 2.1. Let $\Phi: K \rightarrow L$ be u.s.c., and let $k, n \in N$.

A chain mapping

$$
\varphi_{1}: C_{*}(K, l) \longrightarrow C_{*}(L, k) \quad(l \geqq k)
$$

is called $n-k$-approximation of $\Phi$, provided the following condition is satisfied:

For any simplex $\sigma \in \tau^{l}$ there is a point $y(\sigma) \in K$ such that

$$
\begin{aligned}
\sigma & \subset s t^{n}(y(\sigma), k) \\
\operatorname{carr} \varphi_{1} \sigma & \subset s t^{n}(\Phi(y(\sigma)), k) .
\end{aligned}
$$

EXAMPLE 2.2. Let $f: K \rightarrow L$ be continuous and single-valued. Then any chain map $f^{\prime}: C_{*}(K, l) \rightarrow C_{*}(L, k)$ which is induced by a simplicial approximation $\bar{f}$ of $f, \bar{f}:\left(K, \tau^{l}\right) \rightarrow\left(L, \mu^{k}\right)(l \geqq k)$, is a $2-k$ approximation of $f$.

Definition 2.3. Let $\Phi: K \rightarrow L$ be u.s.c. A graded set

$$
A(\Phi)=\left\{A(\Phi)_{j}\right\}_{j \in N},
$$

where $A(\Phi)_{j} \subset \operatorname{hom}\left(C_{*}(K, j), C_{*}(L, j)\right)$, is called approximation system (A-system) for $\Phi$, provided there is an integer $n=n(A(\Phi))$ such that the following properties are satisfied:

(1) $\varphi \in A(\Phi)_{j} \Rightarrow \varphi=\varphi_{1} b$, where $\varphi_{1}$ is a

$n-j$-approximation of $\Phi(b=$ subdivision map).

(2) For all $j \in N$ there is an integer $j_{1}=j_{1}(A(\Phi))$ such that for all $l, m \geqq j_{1}(m \geqq l)$ and for all $\varphi=\varphi_{1} b \in A(\Phi)_{l}, \psi=\psi_{1} b \in A(\Phi)_{m}$

$\varphi_{1} \chi$ and $\chi_{\psi_{1}}$ are homotopic with a small homotopy,

i.e., the diagram

\footnotetext{
1 We assume always $\phi(x) \neq \varnothing$ and closed for all $x \in K$.
} 


$$
\begin{aligned}
C_{*}\left(K, l_{1}\right) & \stackrel{\varphi_{1}}{\longrightarrow} C_{*}(L, l) \\
\chi \uparrow & \uparrow \chi \quad\left(m_{1} \geqq l_{1}\right) \\
C_{*}\left(K, m_{1}\right) & \underset{\psi_{1}}{\longrightarrow} C_{*}(L, m)
\end{aligned}
$$

is homotopy commutative with a chain homotopy $D$ which satisfies the following smallness condition:

For any simplex $\sigma \in \tau^{m_{1}}$ there is a point $z(\sigma) \in K$ such that

$$
\begin{gathered}
\sigma \subset s t^{n}(z(\sigma), j) \\
\operatorname{carr} D \sigma \subset s t^{n}(\Phi(z(\sigma)), j) .
\end{gathered}
$$

EXAMPLE 2.4. Let $f: K \rightarrow L$ be continuous and single-valued. Then, an approximation system for $f$ is obtained in a natural way by simplicial approximation:

$$
\begin{aligned}
& A^{*}(f)=\left\{A^{*}(f)_{j}\right\}_{j \in N}, \quad \text { with } \\
& A^{*}(f)_{j}:=\left\{\varnothing=f^{\prime} b \mid f^{\prime}: C_{*}(K, l) \longrightarrow C_{*}(L, j)\right\}
\end{aligned}
$$

where $f^{\prime}$ is induced by simplicial approximation, see 2.2 . $A^{*}(f)$ is called the approximation system induced by $f$.

Proof. Set $n=n\left(A^{*}(f)\right):=2$, and for $j \in N$ set $j_{1}=j_{1}\left(A^{*}(f)\right):=j$.

Then, it is well known [22, p. 36/37] that $f^{\prime} \chi$ and $\chi f^{\prime \prime}$ are (contiguous) homotopic with a small homotopy, provided $f^{\prime} b \in A^{*}(f)_{l}$, $f^{\prime \prime} b \in A^{*}(f)_{m}$, where $m \geqq l \geqq j$.

The following lemma detects an important relation between $I$ systems and $A$-systems.

LEMMA AND DEFINITION 2.5. Let $U \subset K$ be open and polyhedral, and let $A(\Phi)$ be an $A$-system for $\Phi: K \rightarrow K$.

Then $A(\Phi)$ is an I-system on $U$ provided $(K, \Phi, U)$ is admissible.

This I-system is called the I-system induced by $A(\Phi)$ and denoted by $M(K, U ; A(\Phi))$.

Proof. Without loss of generality we assume $n(A(\Phi))=1$; for $n(A(\Phi))>1$ the same arguments apply. Choose $k \in N$ sufficiently large such that the following boundary condition is satisfied:

$$
y \in K, s t^{3}(y, k) \cap \partial U \neq \varnothing \Longrightarrow s t^{3}(y, k) \cap s t(\Phi(y), k)=\varnothing .
$$

Since $A(\Phi)$ is an $A$-system we obtain for $j=k$ an integer $j_{1}=$ $j_{1}(A(\Phi))$.

We claim that $A(\Phi)$ is an $I$-system on $U$ with $k_{0}=$ $k_{0}\left(M(K, U ; A(\Phi)):=j_{1}\right.$ : 
Therefore let $l, m \in N, m \geqq l \geqq k_{0}$, and let

$$
\varphi=\varphi_{1} b \in A(\Phi)_{l}, \quad \psi=\psi_{1} b \in A(\Phi)_{m} .
$$

We have to show that $\varphi$ and $\chi_{\psi b} b$ are admissible homotopic, see 1.1 .

$$
\begin{aligned}
& C_{*}(\bar{U}, l) \stackrel{b}{\longrightarrow} C_{*}\left(\bar{U}, l_{1}\right) \stackrel{\varphi_{1}}{\longrightarrow} C_{*}(K, l) \\
& C_{*}(\bar{U}, m) \underset{b}{\stackrel{\ddots}{\longrightarrow}} C_{*}\left(\bar{U}, m_{1}\right) \underset{\psi_{1}}{\longrightarrow} C_{*}(K, m)
\end{aligned}
$$

Diagram (I) is commutative, and diagram (II) is homotopy commutative with a small homotopy $D$. Thus, $\varphi$ and $\chi_{\psi} b$ are homotopic with the homotopy $D_{1}:=D b$.

We prove that $D_{1}$ satisfies the boundary condition of an $I$-system:

Assuming that $D_{1}$ does not satisfy the boundary condition we have that there exists a simplex $\sigma \in \tau^{l}$ such that

$$
\begin{aligned}
& s t(\sigma, l) \cap \partial U \neq \varnothing \text { and } \\
& s t(\sigma, l) \cap \operatorname{carr} D_{1} \sigma \neq \varnothing .
\end{aligned}
$$

Hence, there is a simplex $\sigma_{1} \in \tau^{l}$ such that

$$
\sigma_{1} \subset \operatorname{st}(\sigma, l) \text { and } \sigma_{1} \subset \operatorname{carr} D_{1} \sigma .
$$

Let $\sigma_{2}$ be a simplex in $\tau^{m_{1}}$ such that

$$
\sigma_{2} \in b \sigma \text { and } \sigma_{1} \subset \operatorname{carr} D \sigma_{2} \text {. }
$$

Since the homotopy $D$ is small, there is a point $z\left(\sigma_{2}\right) \in K$ such that

$$
\begin{aligned}
\sigma_{2} & \subset s t\left(z\left(\sigma_{2}\right), k\right) \text { and } \\
\operatorname{carr} D \sigma_{2} & \subset \operatorname{st}\left(\Phi\left(z\left(\sigma_{2}\right)\right), k\right) .
\end{aligned}
$$

Hence,

$$
s t(\sigma, l) \cap \operatorname{st}\left(\Phi\left(z\left(\sigma_{2}\right)\right), k\right) \neq \varnothing .
$$

Because of $\sigma_{2} \subset \sigma$, and, thus, $s t^{2}\left(z\left(\sigma_{2}\right), k\right) \supset \sigma$, we see that

$$
s t^{3}\left(z\left(\sigma_{2}\right), k\right) \cap s t\left(\Phi\left(z\left(\sigma_{2}\right)\right), k\right) \neq \varnothing
$$

and

$$
s t^{3}\left(z\left(\sigma_{2}\right), k\right) \cap \partial U \neq \varnothing
$$

which is impossible because of the choice of $k$. Thus, $D_{1}$ is admissible. 
That $\left.\psi b \chi\right|_{\bar{U}}$ and $\psi$ are admissible homotopic is proved in a similar way.

\section{Fixed point index for multivalued mappings.}

DeFinition 2.6. Let $U \subset K$ be open and polyhedral, and let $\Phi: K \rightarrow K$ be u.s.c.. Let $(K, \Phi, U)$ be admissible, and let $A=A(\Phi)$ be an $A$-system for $\Phi$. Then

$$
I_{A}(K, \Phi, U):=I(M)(K, U ; A(\Phi)) \in \boldsymbol{F}
$$

is called the fixed point index of $\Phi$ in $U$ (with resp. to $A$ ).

In the following proposition we list some properties of the fixed point index.

Lemma 2.7. Let $\Phi_{1}: K \rightarrow L$ and $\Phi_{2}: L \rightarrow K$ be u.s.c., and let $A\left(\Phi_{1}\right), A\left(\Phi_{2}\right)$ be A-systems for $\Phi_{1}$ and $\Phi_{2}$. Let $C \subset K$ be a closed subset of $K$ such that for all $x \in C, x \notin \Phi_{2} \Phi_{1}(x)$.

There is an integer $l_{0} \in N$ such that for all $l \geqq l_{0}$ and for all $\varphi \in A\left(\Phi_{1}\right)_{l}, \psi \in A\left(\Phi_{2}\right)_{l}$ the following property holds:

For any simplex $\sigma \in \tau^{l}$ with $\sigma \subset \operatorname{st}\left(C, l_{0}\right)$ and for any simplex $\bar{\sigma} \in \varphi(\sigma)$

$$
\sigma \notin \psi(\bar{\sigma})
$$

Proof. Without loss of generality we assume $n\left(A\left(\Phi_{1}\right)\right)=$ $n\left(A\left(\Phi_{2}\right)\right)=1$.

Since $\Phi_{1}$ and $\Phi_{2}$ are u.s.c., and since $C$ is compact, there is $k \in N$ such that

$$
s t\left(\Phi_{2}\left(s t^{2}\left(\Phi_{1}(s t(\sigma, k)), k\right)\right), k\right) \cap s t(\sigma, k)=\varnothing
$$

for all simplices $\sigma \subset s t(C, k)$.

For $j=k$ we find integers $j_{1}=j_{1}\left(A\left(\Phi_{1}\right)\right), j_{1}^{\prime}=j_{1}\left(A\left(\Phi_{2}\right)\right)$. Then the lemma follows with $l_{0}:=\max \left\{j_{1}, j_{1}^{\prime}\right\}$.

Proposition 2.8 (Additivity). Let $U \subset K$ be open and polyhedral, and let $\Phi: K \rightarrow K$ be u.s.c.. hedral.

Let $U_{1}, U_{2} \subset U$ be open, disjoint subsets of $U$ which are polyAssume that the fixed point set of $\left.\Phi\right|_{\bar{U}}$ is contained in $U_{1} \cup U_{2}$,

$$
\operatorname{Fix}\left(\left.\Phi\right|_{\bar{U}}\right) \subset U_{1} \cup U_{2} .
$$

Let $A=A(\Phi)$ be an $A$-system for $\Phi$. 
Then

$$
I_{A}(K, \Phi, U)=I_{A}\left(K, \Phi, U_{1}\right)+I_{A}\left(K, \Phi, U_{2}\right)
$$

Proof. The proof follows immediately from 2.7 and 1.3 .

Beside the ADDITIVITY we obtain (with the same arguments) an excision property which allows to define the fixed point index on arbitrary open sets.

Proposition 2.9 (Excision). Let $U_{1} \subset U \subset K$ be open and polyhedral, and let $\Phi: K \rightarrow K$ be u.s.c.. Let $A=A(\Phi)$ be an A-system for $\Phi$. If $\operatorname{Fix}\left(\left.\Phi\right|_{\bar{U}}\right) \subset U_{1}$, then

$$
I_{A}(K, \Phi, U)=I_{A}\left(K, \Phi, U_{1}\right) .
$$

Definition 2.10. Let $V \subset K$ be open, and let $\Phi: K \rightarrow K$ be u.s.c..

Let $(K, \Phi, V)$ be admissible, and let $U \subset V$ be open and polyhedral such that $\operatorname{Fix}\left(\left.\Phi\right|_{\vec{V}}\right) \subset U$. Let $A=A(\Phi)$ be an $A$-system for $\Phi$.

$$
I_{A}(K, \Phi, V):=I_{A}(K, \Phi, U) .
$$

Observe that ADDITIVITY and EXCISION generalize immediately to this fixed point index on open sets.

EXAMPLE 2.11. Let $f: K \rightarrow K$ be continuous and single-valued, and let $A^{*}(f)$ be the $A$-system induced by $f$, see 2.4. Then Definition 2.10 of fixed point index coincides with the definition given by O'Neill in [34]:

$$
I_{A^{*}}(K, f, V)=i\left(K, f, V_{1}\right)
$$

\section{Homotopy of $A$-systems.}

DEFINITION 2.12. Let $U \subset K$ be open and polyhedral, and let $\Phi_{1}, \Phi_{2}: K \rightarrow L$ be u.s.c. . Let $H: \bar{U} \times I \rightarrow L$ be an u.s.c. homotopy joining $\left.\Phi_{1}\right|_{\bar{v}}$ and $\left.\Phi_{2}\right|_{\bar{U}} . \quad(I=[0,1])$ Let $A\left(\Phi_{1}\right), A\left(\Phi_{2}\right)$ be $A$-systems for $\Phi_{1}, \Phi_{2} . \quad A\left(\Phi_{1}\right)$ and $A\left(\Phi_{2}\right)$ are called H-homotopic provided there is an integer $m \in N$ such that the following property is satisfied:

For all $j \in N$ there is an integer $j_{2} \in N$ such that for all $l \geqq j_{2}$ there are maps

$$
\varphi=\varphi_{1} b \in A\left(\Phi_{1}\right)_{l}, \quad \psi=\psi_{1} b \in A\left(\Phi_{2}\right)_{l},
$$

where

such that

$$
\varphi_{1}, \psi_{1}: C_{*}\left(K, l_{1}\right) \longrightarrow C_{*}(K, l) \quad\left(l_{1} \geqq l\right)
$$




$$
\bar{\varphi}_{1}:=\left.\varphi_{1}\right|_{C_{*}\left(\bar{U}, l_{1}\right)} \text { and } \bar{\psi}_{1}:=\left.\psi_{1}\right|_{C_{*}\left(\vec{U}, l_{1}\right)}
$$

are homotopic with an homotopy $D$ satisfying the following condition, for any simplex $\sigma \in \tau^{l_{1}}$ there is a point $d(\sigma) \in K$ such that

$$
\begin{gathered}
\sigma \subset s t^{m}(d(\sigma), j) \\
\operatorname{carr} D \sigma \subset s t^{m}(H(d(\sigma) \times I), j) .
\end{gathered}
$$

EXAMPLE 2.13. Let $f_{1}, f_{2}: K \rightarrow L$ be continuous and single-valued, and let $A^{*}\left(f_{1}\right), A^{*}\left(f_{2}\right)$ be the $A$-systems induced by $f_{1}, f_{2}$. Let $U \subset K$ be open and polyhedral, and let $H: \bar{U} \times I \rightarrow L$ be a homotopy joining $\left.f_{1}\right|_{\bar{U}}$ and $\left.f_{2}\right|_{\bar{U}}$. Then, $A^{*}\left(f_{1}\right)$ and $A^{*}\left(f_{2}\right)$ are $H$-homotopic.

Proof. Define

$$
\begin{aligned}
K_{1}: & =K \times\{0\} \cup \bar{U} \times I \cup K \times\{1\} \\
\tau_{1}: & =\tau \times\{0\} \cup(\tau \times I) \cup \tau \times\{1\} .
\end{aligned}
$$

(By $\tau \times I$ we mean a product triangulation $\tau \times \rho$, where $\rho$ is a triangulation of $I$.)

Let $H_{1}: K_{1} \rightarrow L$ be the extension of $H$ with

$$
\left.H_{1}\right|_{K \times\{0\}}=f_{1},\left.\quad H_{1}\right|_{K \times\{1\}}=f_{2} .
$$

Let $j \in N$. Set $j_{2}:=j$.

Choose $H_{1}^{\prime} b \in A^{*}\left(H_{1}\right)_{l}\left(l \geqq j_{2}\right)$, and set

$$
\varphi:=\left.H_{1}^{\prime} b\right|_{\left.C_{*}(K \times(0\})\right)}, \quad \psi:=\left.H_{1}^{\prime} b\right|_{C_{*}(K \times\{1\})},
$$

and $D \sigma:=H_{1}^{\prime} b(\sigma \times I)\left(\sigma \times I\right.$ chain in $\left.(\tau \times I)^{l}\right)$. Thus, $A^{*}\left(f_{1}\right)$ and $A^{*}\left(f_{2}\right)$ are $H$-homotopic with $m=2$.

LEMMA 2.14. Let $U \subset K$ be open and polyhedral, and let $\Phi_{1}, \Phi_{2}$ : $K \rightarrow K$ be u.s.c.. Let $A\left(\Phi_{1}\right), A\left(\Phi_{2}\right)$ be A-systems for $\Phi_{1}, \Phi_{2}$ which are $H$-homotopic.

If $\left(K, H_{t}, U\right)$ is admissible for all $t \in I$, then the induced $I-$ systems $M\left(K, U ; A\left(\Phi_{1}\right)\right)$ and $M\left(K, U ; A\left(\Phi_{2}\right)\right)$ are homotopic $\left(H_{t}(x):=\right.$ $H(x, t))$.

Proof. We assume without loss of generality $n\left(A\left(\Phi_{1}\right)\right)=$ $n\left(A\left(\Phi_{2}\right)\right)=1$. Choose $k \in N$ sufficiently large such that for all $(z, t) \in \bar{U} \times I$,

$$
s t^{3}(z, k) \cap \partial U \neq \varnothing \Longrightarrow s t^{3}(z, k) \cap s t(H(z, t), k)=\varnothing .
$$

For $j=k$ we obtain from 2.12 an integer $j_{2}$ such that for all $l \geqq$ $j_{2}$ there are maps 


$$
\varphi=\varphi_{1} b \in A\left(\Phi_{1}\right)_{l}, \quad \psi=\psi_{1} b \in A\left(\Phi_{2}\right)_{l}
$$

such that the restrictions $\bar{\varphi}_{1}$ and $\bar{\psi}_{1}$ are homotopic with an homotopy $D$ satisfying the properties described in 2.12 .

Thus, the restrictions $\bar{\varphi}$ and $\bar{\psi}$ are homotopic with $D b$.

The proof that $\bar{\phi}$ and $\bar{\psi}$ are admissible homotopic is similar to the proof of 2.5 , and is therefore omitted.

Proposition 2.15 (Homotopy Invariance). Let $V \subset K$ be open, and let $\Phi_{1}, \Phi_{2}: K \rightarrow K$ be u.s.c.. Let $H: \bar{V} \times I \rightarrow K$ be an u.s.c. homotopy joining $\left.\Phi_{1}\right|_{\bar{V}}$ and $\left.\Phi_{2}\right|_{\bar{V}}$, such that $\left(K, H_{t}, V\right)$ is admissible for all $t \in I$. Let $A_{1}=A\left(\Phi_{1}\right), A_{2}=A\left(\Phi_{2}\right)$ be A-systems for $\Phi_{1}, \Phi_{2}$, which are H-homotopic on $U$, where $U \subset V$ is an open, polyhedral subset such that $\bar{V} \backslash U$ contains no fixed points of $H_{t}, t \in I$.

Then,

$$
I_{A_{1}}\left(K, \Phi_{1}, V\right)=I_{A_{2}}\left(K, \Phi_{2}, V\right)
$$

Proof. The proof follows immediately from 2.14 and 1.5.

\section{Commutativity and mod-p property.}

Lemma 2.16. Let $\Phi_{1}: K \rightarrow L, \Phi_{2}: L \rightarrow M$ be u.s.c., and let $A\left(\Phi_{1}\right)$, $A\left(\Phi_{2}\right)$ be A-systems for $\Phi_{1}, \Phi_{2}$. Then $A:=A\left(\Phi_{2}\right) \circ A\left(\Phi_{1}\right)$ is an A-system for $\Phi_{2} \Phi_{1}$.

Proof. We assume without loss of generality

$$
n\left(A\left(\Phi_{1}\right)\right)=n\left(A\left(\Phi_{2}\right)\right)=1 .
$$

Let $j \in N$. Then for every $y \in K$ there is $j(y) \in N$ such that

$$
\Phi_{2}\left(s t^{3}\left(\Phi_{1}(y), j(y)\right)\right) \subset s t\left(\Phi_{2} \Phi_{1}(y), j\right) .
$$

Since $\Phi_{1}$ is u.s.c. and $K$ is compact we find an integer $j^{\prime} \in N, j^{\prime} \geqq j$, such that for every $y \in K$ there is a point $u=u(y) \in K$ such that

$$
\begin{aligned}
& y \in s t(u, j) \\
& s t^{2}\left(\Phi_{1}(y), j^{\prime}\right) \subset s t^{3}\left(\Phi_{1}(u), j(u)\right),
\end{aligned}
$$

and therefore,

$$
\Phi_{2}\left(s t^{2}\left(\Phi_{1}(y), j^{\prime}\right)\right) \subset \operatorname{st}\left(\Phi_{2} \Phi_{1}(u), j\right) .
$$

For $j^{\prime}$ we obtain by 2.3 an integer $j_{1}=j_{1}^{\prime}\left(A\left(\Phi_{1}\right)\right)$ and an integer $j_{2}=j_{1}^{\prime}\left(A\left(\Phi_{2}\right)\right)$. Let $l, m \geqq \max \left\{j_{1}, j_{2}\right\}$. 


$$
\begin{aligned}
& C_{*}\left(K, l_{2}\right) \stackrel{\varphi_{1}}{\longrightarrow} C_{*}(L, l) \stackrel{b}{\longrightarrow} C_{*}\left(L, l_{1}\right) \stackrel{\varphi_{1}^{\prime}}{\longrightarrow} C_{*}(M, l)
\end{aligned}
$$

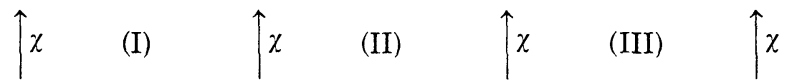

$$
\begin{aligned}
& C_{*}\left(K, m_{2}\right) \underset{\psi_{1}}{\longrightarrow} C_{*}(L, m) \underset{b}{\longrightarrow} C_{*}\left(L, m_{1}\right) \underset{\psi_{1}^{\prime}}{\longrightarrow} C_{*}(M, m) \\
& \varphi=\varphi_{1} b \in A\left(\Phi_{1}\right)_{l}, \quad \varphi^{\prime}=\varphi_{1}^{\prime} b \in A\left(\Phi_{2}\right)_{l} \\
& \psi^{\prime}=\psi_{1} b \in A\left(\Phi_{1}\right)_{m}, \quad \psi^{\prime}=\psi_{1}^{\prime} b \in A\left(\Phi_{2}\right)_{m} \text {. }
\end{aligned}
$$

We show that $\varphi_{1}^{\prime} b \varphi_{1} \chi$ and $\chi_{\psi_{1}^{\prime}}^{\prime} b \psi_{1}^{\prime}$ are homotopic with a small homotopy.

Diagram (I) is homotopic commutative with a small homotopy $D_{1}$.

Diagram (III) is homotopic commutative with a small homotopy $D_{3}$.

Diagram (II) is homotopic commutative with a homotopy $D_{2}$ satisfying: $\operatorname{carr} D_{2} \sigma \subset \operatorname{carr} \chi(\sigma)$, for $\sigma \in \mu^{m}$.

Hence, $\varphi^{\prime} \varphi_{1} \chi$ and $\chi_{\psi^{\prime}} \psi_{1}$ are homotopic with the homotopy

$$
\begin{aligned}
D:=\Delta_{1}+\Delta_{2}+\Delta_{3}, & \text { where } \Delta_{1}=\varphi_{1}^{\prime} b D_{1}, \Delta_{2}=\varphi_{1}^{\prime} D_{2} \psi_{1} \\
\text { and } \Delta_{3} & =D_{3} b \psi_{1} .
\end{aligned}
$$

We prove the smallness condition for $\Delta_{1}$ (the others being similar):

For a simplex $\sigma \in \tau^{m_{2}}$ by 2.3 there is a point $z_{1}(\sigma) \in K$ such that

$$
\begin{aligned}
\sigma & \subset \operatorname{st}\left(z_{1}(\sigma), j^{\prime}\right) \\
\operatorname{carr} D_{1} \sigma & \subset \operatorname{st}\left(\Phi_{1}\left(z_{1}(\sigma)\right), j^{\prime}\right)
\end{aligned}
$$

By choice of $j^{\prime}$ for the point $z_{1}(\sigma)$ we can find a point $u=u\left(z_{1}(\sigma)\right)$ such that

$$
\begin{aligned}
& z_{1}(\sigma) \in s t(u, j) \\
& \Phi_{2}\left(s t^{2}\left(\Phi_{1}\left(z_{1}(\sigma)\right), j^{\prime}\right)^{2} \subset s t\left(\Phi_{2} \Phi_{1}(u), j\right) .\right.
\end{aligned}
$$

Define $z(\sigma):=u\left(z_{1}(\sigma)\right)$.

Then a straightforward calculation gives

$$
\begin{aligned}
\sigma & \subset s t^{2}(z(\sigma), j) \\
\operatorname{carr} \Delta_{1}(\sigma) & \subset s t^{2}\left(\Phi_{2} \Phi_{1}(z(\sigma)), j\right) .
\end{aligned}
$$

Thus, $D$ satisfies the smallness condition, and $A$ is an $A$-system for $\Phi_{2} \Phi_{1}$ with $n(A)=2$.

Proposition 2.17 (Commutativity). Let $W \subset K$ be open, and let $\Phi_{1}: K \rightarrow L, \Phi_{2}: L \rightarrow K$ be u.s.c.. Let $A_{1}=A\left(\Phi_{1}\right), A_{2}=A\left(\Phi_{2}\right)$ be $A$ systems for $\Phi_{1}, \Phi_{2}$. Assume that $\left(K, \Phi_{2} \Phi_{1}, W\right)$ and $\left(L, \Phi_{1} \Phi_{2}, \Phi_{2}^{-1}(W)\right)$ are admissible, and that the following condition is satisfied: 


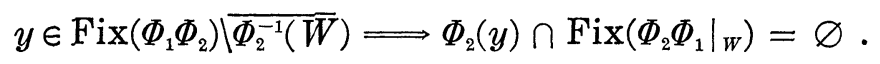

Then

$$
I_{A_{1} A_{2}}\left(L, \Phi_{1} \Phi_{2}, \Phi_{2}^{-1}(W)\right)=I_{A_{2} A_{1}}\left(K, \Phi_{2} \Phi_{1}, W\right)
$$

Proof. We assume without loss of generality $n\left(A_{1}\right)=n\left(A_{2}\right)=1$. Define

$$
\begin{aligned}
& B_{1}:=\Phi_{2}^{-1}(W) \cap \operatorname{Fix}\left(\Phi_{1} \Phi_{2}\right) \\
& B_{2}:=\operatorname{Fix}\left(\Phi_{1} \Phi_{2}\right) \backslash \Phi_{2}^{-1}(W) \\
& B_{3}:=\operatorname{Fix}\left(\Phi_{2} \Phi_{1}\right) \cap W .
\end{aligned}
$$

There is an integer $k_{1} \in N$ such that $s t^{2}\left(\Phi_{2}\left(B_{1}\right), k_{1}\right) \subset W$.

Let $O \subset L$ be an open set such that

$$
B_{1} \subset \mathcal{O}, \quad \Phi_{2}(\mathcal{O}) \subset S t\left(\Phi_{2}\left(B_{1}\right), k_{1}\right) .
$$

There is an integer $l_{1} \in N$ such that $\operatorname{st}^{2}\left(B_{1}, l_{1}\right) \subset \mathcal{O}$.

Define

$$
V:=\operatorname{int} s t\left(B_{1}, l_{1}\right) \text {. }
$$

Since $\Phi_{2}\left(B_{2}\right) \cap B_{3}=\varnothing$, there is $k_{2} \geqq k_{1}$ such that

$$
s t^{2}\left(\Phi_{2}\left(B_{2}\right), k_{2}\right) \cap s t\left(B_{3}, k_{2}\right)=\varnothing \text {. }
$$

Define

$$
U:=\operatorname{int} \operatorname{st}\left(B_{3}, k_{2}\right) \subset W .
$$

There is an open set $\mathscr{O}_{1} \supset B_{2}$ such that

$$
\mathscr{O}_{1} \cap \overline{\Phi_{2}^{-1}(W)}=\varnothing, \Phi_{2}\left(\mathcal{O}_{1}\right) \subset \operatorname{st}\left(\Phi_{2}\left(B_{2}\right), k_{2}\right) \text {. }
$$

Therefore, there exists $l_{2} \in N, l_{2} \geqq l_{1}$ such that

$$
s t^{2}\left(B_{2}, l_{2}\right) \subset \mathscr{O}_{1} \text {. }
$$

Define $V_{1}:=\operatorname{st}\left(B_{2}, l_{2}\right), V_{2}:=L \backslash V_{1}$.

Since for all $x \in C:=\overline{W \backslash U}, x \notin \Phi_{2} \Phi_{1}(x)$ we obtain from 2.7 an integer $l_{0}$, and since for all $x \in C^{\prime}:=\overline{V_{2} \backslash V}, x \notin \Phi_{1} \Phi_{2}(x)$, we obtain from 2.7 an integer $l_{0}^{\prime}\left(l_{0} \geqq k_{2}, l_{0}^{\prime} \geqq l_{2}\right)$.

Set $j:=\max \left\{l_{0}, l_{0}^{\prime}\right\}$, and define $\tilde{k}:=\max \left\{j_{1}\left(A_{1}\right), j_{1}\left(A_{2}\right)\right\}$. Thus, the assumption of 1.6 is satisfied, and we obtain

$$
I_{A_{2} A_{1}}\left(K, \Phi_{2} \Phi_{1}, U\right)=I_{A_{1} A_{2}}\left(L, \Phi_{1} \Phi_{2}, V\right) \text {. }
$$

ExAmple 2.18. Let $W \subset K$ be open, and let $f: K \rightarrow L, g: L \rightarrow K$ 
be continuous and single-valued such that $(K, g f, W)$ is admissible. Then

$$
i(K, g f, W)=i\left(L, f g, g^{-1}(W)\right)
$$

Proof. Let $A_{1}=A^{*}(f), A_{2}=A^{*}(g)$ be the $A$-systems induced by $f$ and $g$. Then by 2.17

$$
I_{A_{2} A_{1}}(K, g f, U)=I_{A_{1} A_{2}}(L, f g, V) .
$$

( $U$ and $V$ are chosen as in the proof of 2.17.) Since $A^{*}(g f)_{k} \cap$ $\left(A_{2} \circ A_{1}\right)_{k} \neq \varnothing$ and $A^{*}(f g)_{k} \cap\left(A_{1} \circ A_{2}\right)_{k} \neq \varnothing$ for all $k \in N$, this implies

$$
i(K, g f, W)=i\left(L, f g, g^{-1}(W)\right) .
$$

Proposition 2.19 (Mod-p Property). Let $F=Z_{p}, p$ prime. Let $W \subset K$ be open, and let $\Phi: K \rightarrow K$ be u.s.c., such that $(K, \Phi, W)$ and $\left(K, \Phi^{p}, W\right)$ are admissible. Assume the following condition is satisfied:

$$
y \in \operatorname{Fix}\left(\Phi^{p}\right) \backslash W \Longrightarrow \Phi^{k}(y) \cap \operatorname{Fix}\left(\left.\Phi^{p}\right|_{\bar{W}}\right)=\varnothing \text { for } 1 \leqq k<p .
$$

Let $A=A(\Phi)$ be an A-system for $\Phi$. Then

$$
I_{A}(K, \Phi, W)=I_{A^{p}}\left(K, \Phi^{p}, W\right) \quad(\text { in } F)
$$

$\left(A^{p}=A \circ \cdots \circ A, p\right.$ times $)$.

Proof. Without loss of generality we assume $n(A)=1$. Moreover, we give a proof only for $p=2$, for $p>2$ the proof runs along the same lines.

Define $B_{1}:=\operatorname{Fix}\left(\Phi^{2}\right) \cap W, B_{2}:=\operatorname{Fix}\left(\Phi^{2}\right) \backslash W$.

Since $\Phi\left(B_{2}\right) \cap B_{1}=\varnothing$, there exists $k_{1} \in N$ such that

$$
s t^{2}\left(\Phi\left(B_{2}\right), k_{1}\right) \cap s t\left(B_{1}, k_{1}\right)=\varnothing \text {. }
$$

Define

$$
U:=\operatorname{int} \operatorname{st}\left(B_{1}, k_{1}\right)
$$

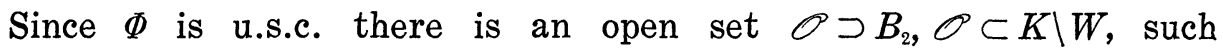
that

$$
\Phi(\mathscr{O}) \subset \operatorname{st}\left(\Phi\left(B_{2}\right), k_{1}\right) .
$$

There exists $k_{2} \geqq k_{1}$ such that $s t^{2}\left(B_{2}, k_{2}\right) \subset \mathcal{O}$. Define

$$
V_{1}:=\operatorname{st}\left(B_{2}, k_{2}\right) \text {. }
$$


Since for all $x \in C:=\overline{K \backslash V_{1} \backslash U}, x \notin \Phi^{2}(x)$, we obtain from 2.7 an integer $l_{0} \geqq k_{2}$. Set $j=l_{0}$ and define

$$
\bar{k}:=j_{1}(A) \text {. }
$$

Then the assumptions of 1.7 are satisfied, and we obtain

$$
I_{A}(K, \Phi, U)=I_{A^{2}}\left(K, \Phi^{2}, U\right) \quad\left(\text { in } Z_{2}\right) .
$$

EXAMPLE 2.20. Let $W \subset K$ be open, and let $f: K \rightarrow K$ be continuous and single-valued. Let $(K, f, W)$ and $\left(K, f^{p}, W\right)$ be admissible, $p$ prime, and assume that for all $x \in \operatorname{Fix}\left(\left.f^{p}\right|_{W}\right)$,

$$
x, f(x), \cdots, f^{p-1}(x) \in W .
$$

Then

$$
i(K, f, W)=i\left(K, f^{p}, W\right) \quad\left(\text { in } Z_{p}\right)
$$

Proof. Let $A=A^{*}(f)$ be the $A$-system induced by $f$. Then, by 2.19

$$
I_{A}(K, f, W)=I_{A^{p}}\left(K, f^{p}, W\right)
$$

Since

$$
\left(A^{*}(f)\right)_{k}^{p} \cap A^{*}\left(f^{p}\right)_{k} \neq \varnothing \quad \text { for all } k \in N
$$

this implies

$$
i(K, f, W)=i\left(K, f^{p}, W\right) \quad\left(\text { in } Z_{p}\right)
$$

REMARK 2.21. (1) The mod-p result of 2.20 was announced in 1971 by Zabrelko and Krasnosel'skil, however they gave only a sketch of proof [45]. A first complete proof (independent of [45]) was presented by Steinlein in [40]. Steinleins proof is of analytic nature and rather complicated. In the meantime, several proofs for the mod- $p$ result (for single-valued maps) appeared [16], [25], [41], [42], [43], but all these proofs are based on certain transversality arguments. Thus, they can not be generalized to multivalued mappings.

(2) The essential ingredients for our proofs in Chapter II are a "simplicial structure" of the spaces and barycentric subdivision. These ingredients were axiomatized by Browder [5] in order to define 'semi-complexes'. Thus, our results should generalize to Browder's category of semi-complexes.

III. Existence of $A$-systems. In this chapter we construct $A$ - 
systems for certain classes of multivalued mappings $\Phi: K \rightarrow L$. It turns out that the existence of an $A$-system for acyclic maps isimplicitly-contained in Vietoris' (and Begle's) proof for the VietorisBegle mapping theorem [1], [44]. Also Eilenberg and Montgomery used chain approximations in their proof of the Eilenberg-Montgomery fixed point theorem [12].

In [35] O'Neill gave a construction of chain approximations for continuous (=upper-and lower semi continuous) multivalued mappings $\Phi$ such that $\Phi(x)$ is acyclic or consists of $n$ acyclic components ( $n$ fixed) for all $x$. Moreover, he proved an "abstract" Lefschetz fixed point theorem for this class of mappings. Our approach of constructing $A$-systems is similar to $0^{\prime}$ Neills approach in [35]. However, we do not need continuity but upper semi continuity for the mapping. The basic ingredients for constructing $A$-systems are based more or less on Vietoris' work.

o. Preliminaries. In this paragraph $H_{*}$ will denote Čech homology with coefficients in a field $F$, [13].

Let $X, Y$ be compact Hausdorff spaces.

Definition 3.1. Let $\Phi: X \rightarrow Y$ be a multivalued map. $\Phi$ is called acyclic (more precisely, $\boldsymbol{F}$-acylic) provided

(i) $\Phi$ is u.s.c..

(ii) $\widetilde{H}_{*}(\Phi(x))=0$ for all $x \in X(\widetilde{H}=$ reduced homology).

Definition 3.2. Let $p: X \rightarrow Y$ be continuous. $\quad p$ is called Vietoris mapping (more precisely, $\boldsymbol{F}$-Vietoris mapping) provided

(i) $p$ is onto

(ii) $p^{-1}(y)$ is acyclic for all $y \in Y$.

REMARK 3.3. Let $\Phi: X \rightarrow Y$ be acyclic, let

$$
\Gamma(\Phi):=\{(x, y) \in X \times Y \mid y \in \Phi(x)\}
$$

be the graph of $\Phi$, and let

$$
\begin{aligned}
& X \stackrel{p}{\longleftarrow} \Gamma(\Phi) \stackrel{q}{\longrightarrow} Y \\
& x \stackrel{\longleftrightarrow}{\longleftrightarrow}(x, y) \longmapsto y
\end{aligned}
$$

be the natural projections.

Then, $\Gamma(\Phi)$ is compact, and $p$ is a Vietoris map.

Definition 3.4. Let $\Phi: X \rightarrow Y$ be acyclic, and let $p, q$ be the natural projections.

Because of the Vietoris-Begle mapping theorem [1], [44] the map $p_{*}: H_{*}(\Gamma(\Phi)) \rightarrow H_{*}(X)$ is an isomorphism. 
The map

$$
\Phi_{*}:=q_{*} p_{*}^{-1}: H_{*}(X) \longrightarrow H_{*}(Y)
$$

is called the induced homomorphism of $\Phi$.

1. Acyclic mappings and $A$-systems. In the following $(K, \tau)$ and $(L, \mu)$ will always denote compact polyhedra with triangulations $\tau$ and $\mu$.

LEMMA AND DEFINITION 3.5. Let $\Phi: K \rightarrow L$ be acyclic, and let $l \in N$. Then, there exists an integer $l_{0} \geqq l$ and a chain map ("l-chain approximation of $\Phi$ ")

$$
\varphi_{1}: C_{*}\left(K, l_{0}\right) \longrightarrow C_{*}(L, l)
$$

with the following properties:

(1) $\varphi_{1}$ is augmentation preserving, i.e., $\varphi_{1}$ maps a vertex into a vertex.

(2) For any simplex $\sigma \in \tau^{l_{0}}$ there is a point $p(\sigma) \in K$ such that

$$
\begin{aligned}
\sigma & \subset s t^{2}(p(\sigma), l) \\
\operatorname{carr} \varphi_{1} \sigma & \subset s t(\Phi(p(\sigma)), l) .
\end{aligned}
$$

Any chain map satisfying (1) and (2) is called an l-chain approximation of $\Phi$.

A proof of 3.5 will be given in the appendix.

REMARK 3.6. In analogy with simplicial approximations of singlevalued mappings chain approximations of $\Phi$ are not unique.

Definition 3.7. Let $\Phi: K \rightarrow L$ be acyclic. Then

$$
A^{*}(\Phi):=\left\{A^{*}(\Phi)_{j}\right\}_{j \in N}
$$

with $A^{*}(\Phi)_{j}:=\left\{\varphi=\varphi_{1} b \mid \varphi_{1} j\right.$-chain approximation of $\left.\Phi\right\}$ is called the A-system induced by $\Phi$.

The following lemma justifies the definition above, i.e., it proves that $A^{*}(\Phi)$ is actually an $A$-system.

LeMma 3.8. Let $\Phi: K \rightarrow L$ be acyclic. For all $j \in N$ there is an integer $j_{1}$ such that for all $l, m \geqq j_{1}(m \geqq l)$ and for all $\varphi=$ $\varphi_{1} b \in A^{*}(\Phi)_{l}, \psi=\psi_{1} b \in A^{*}(\Phi)_{m}$

$$
\varphi_{1} \chi \text { and } \chi_{\psi_{1}} \text { are homotopic with a small homotopy, }
$$


the diagram

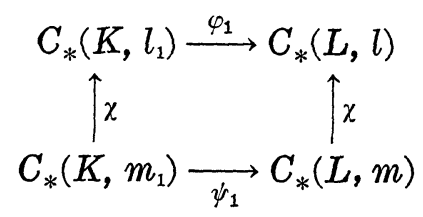

is homotopy commutative with a chain homotopy $D$ satisfying the following smallness condition, for any simplex $\sigma \in \tau^{m_{1}}$ there is a point $c(\sigma) \in K$ such that

$$
\begin{gathered}
\sigma \subset s t^{3}(c(\sigma), j) \\
\operatorname{carr} D \sigma \subset s t(\Phi(c(\sigma)), j) .
\end{gathered}
$$

Lemma 3.8 will be proved in the appendix.

The following lemma is proved in the same way as 3.8 .

Lemma 3.8'. Let $\Phi: K \rightarrow L$ be acyclic. For all $j \in N$ there is an integer $j_{1}$ such that for all $l, m \geqq j_{1}(m \geqq l)$ the following holds:

If $\phi_{1}$ is an $n$-l-approximation (see (2.1)) of $\Phi$, and if $\psi_{1}$ is an $k$-m-approximation of $\Phi$, and if $\varphi_{1}$ and $\psi_{1}$ are augmentation preserving, then

$\varphi_{1} \chi$ and $\chi_{\psi_{1}}$ are homotopic with a small homotopy $D$,

i.e., there is an integer $i \in N$ such that for any simplex $\sigma \in \tau^{m_{1}}$ there is a point $c(\sigma) \in K$ such that

$$
\begin{gathered}
\sigma \subset s t^{i}(c(\sigma), j) \\
\operatorname{carr} D \sigma \subset s t^{i}(\Phi(c(\sigma)), j) .
\end{gathered}
$$

In view of the normalization property the following corollary is important.

Corollary 3.9. Let $\Phi: K \rightarrow L$ be acyclic. There is an integer $k_{0} \in N$ such that for all $k \geqq k_{0}$ and for all $k$-chain approximations $\varphi_{1}$ of $\Phi$ :

$$
\left(\varphi_{1}\right)_{*}=\Phi_{*}: H_{*}(K) \longrightarrow H_{*}(L) \text {. }
$$

Proof. The proof follows from [1] and 3.8.

In the following we list some classes of multivalued mappings which have $A$-systems. 
EXAmple 1. The class $\mathscr{T}$ [38] of all multivalued mappings $\Phi: K \rightarrow L$ which can be "factorized",

$$
\Phi=\Phi_{n} \circ \cdots \circ \Phi_{0},
$$

where $\Phi_{i}: X_{i} \rightarrow X_{i+1}$ are acyclic maps of compact polyhedra $\left(X_{0}=K\right.$, $X_{n+1}=L$ ) has an $A$-system. (One can replace $X_{1}, \cdots, X_{n}$ by compact Hausdorff spaces.)

Example 2. The class $\mathscr{A}$ of all 'admissible' [17] multivalued maps $\Phi: K \rightarrow L$ has an $A$-system.

EXAMPLE 3. The class of all u.s.c. multivalued maps $\Phi: K \rightarrow L$ for which there exists a "single-valued" approximation, i.e., for all $k \in N$ there is a continuous single-valued mapping $f_{k}: K \rightarrow L$ such that

$$
f_{k}(x) \in \operatorname{st}(\Phi(x), k), \text { for all } x \in K \text {, }
$$

has an $A$-system, see e.g., [2], [8], [31], [32].

EXAMPLE 4. It can be shown that the class of all continuous (u.s.c. and l.s.c.) mappings $\Phi: K \rightarrow L$, where $\Phi(x)$ is acyclic or has $n$ acyclic components ( $n$ fixed) [34], has an $A$-system.

REMARK 3.10. Any u.s.c. multivalued mapping for which there exists an $A$-system has an induced homomorphism in homology in the sense of O'Neill [34]. Because there are multivalued mappings which do not have an induced homomorphism, see [34], there are multivalued mappings which do not have an $A$-system.

IV. Fixed point index for acyclic maps. In this chapter we apply the results of Chapters II and III to define a fixed point index for acyclic maps. In the following $(K, \tau)$ and $(L, \mu)$ will always denote compact polyhedra with triangulations $\tau$ and $\mu$.

1. Definition of fixed point index. Call an $A$-system for an u.s.c. mapping $\Phi: K \rightarrow L$ regular if all $\varphi \in A(\Phi)_{k}, k \in N$, are augmentation preserving.

Proposition 4.1. Let $U \subset K$ be open and polyhedral, and let $\Phi: K \rightarrow K$ be acyclic. Let $(K, \Phi, U)$ be admissible. Let further $A_{1}=$ $A_{1}(\Phi), A_{2}=A_{2}(\Phi)$ be two regular A-systems for $\Phi$.

Then, the induced I-systems $M\left(K, U ; A_{1}\right)$ and $M\left(K, U ; A_{2}\right)$ are homotopic. 
Proof. Follows from 3.8'.

Definition 4.2. Let $U \subset K$ be open, and let $\Phi: K \rightarrow K$ be acyclic such that $(K, \Phi, U)$ is admissible. Then

$$
i(K, \Phi, U):=I_{\Delta}(K, \Phi, U) \in \boldsymbol{F},
$$

where $A=A(\Phi)$ is any regular $A$-system for $\Phi$, is called the fixed point index of $\Phi$ on $U$. Because of 4.1 and 1.5 the definition of $i(K, \Phi, U)$ is independent of the choice of $A(\Phi)$.

2. Properties of the fixed point index. In order to obtain homotopy invariance for our fixed point index we need a lemma.

LEMmA 4.3. Let $U \subset K$ be open and polyhedral. Let $\Phi_{1}, \Phi_{2}: K \rightarrow$ $L$ be acyclic, and assume that $\left.\Phi_{1}\right|_{\bar{U}}$ and $\left.\Phi_{2}\right|_{\bar{U}}$ are homotopic with an acyclic homotopy $F: \bar{U} \times I \rightarrow L$. Then, for all $l \in N$ there is $l_{0} \in N$ such that for all $k \geqq l_{0}$ there are maps $\varphi=\varphi_{1} b \in A^{*}\left(\Phi_{1}\right)_{k}, \psi=$ $\psi_{1} b \in A^{*}\left(\Phi_{2}\right)_{k}$,

$$
\varphi_{1}, \psi_{1}: C_{*}\left(K, k_{0}\right) \longrightarrow C_{*}(L, k)
$$

such that $\bar{\varphi}_{1}:=\left.\varphi_{1}\right|_{c_{*}\left(\bar{U}, k_{0}\right)}$ and $\bar{\psi}_{1}:=\left.\psi_{1}\right|_{C_{*}\left(\bar{U}, k_{0}\right)}$ are homotopic with a chain homotopy $D$ satisfying the following property, for any simplex $\sigma \in \tau^{k_{0}}, \sigma \subset \bar{U}$ there is a point $d(\sigma) \in K$ such that

$$
\begin{gathered}
\sigma \subset s t^{2}(d(\sigma), l) \\
\operatorname{carr} D \sigma \subset s t^{2}(F(d(\sigma) \times I), l) .
\end{gathered}
$$

Proof. Let $l \in N$. For $x \in \bar{U}$ there is an open neighborhood $\mathscr{O}_{x}$ such that $F\left(\mathcal{O}_{x} \times I\right) \subset s t(F(x \times I), l)$. We can assume that the covering $\left\{\mathcal{O}_{x}\right\}_{x \in \bar{U}}$ is a refinement of the star covering $\operatorname{st}\left(\tau^{l}\right)$. Let $\omega_{1}:=\left\{\mathscr{O}_{x_{i}}\right\}_{i=1}^{s}$ be a finite subcovering of $\bar{U}$.

Then there is $l_{0} \in N$ such that

$$
\left\{\text { int } s t^{2}\left(\sigma, l_{0}\right) \mid \sigma \in \tau^{l_{0}}\right\}
$$

is a refinement of $\omega_{1}$.

Set

$$
\begin{aligned}
& K_{1}=K \times\{0\} \cup \bar{U} \times I \cup K \times\{1\} \\
& \tau_{1}:=\tau \times\{0\} \cup \tau \times I \cup \tau \times\{1\},
\end{aligned}
$$

and let $F_{1}: K_{1} \rightarrow L$ be the extension of $F$ such that $\left.F_{1}\right|_{K \times\{0\}}=\Phi_{1}$, $\left.F_{1}\right|_{K \times\{1\}}=\Phi_{2}$.

Choose $k \geqq l_{0}$. 
Because of 3.5 there is a $k$-chain approximation of $F_{1}$,

$$
\vartheta_{1}: C_{*}\left(K_{1}, k_{0}\right) \longrightarrow C_{*}(L, k) \text {. }
$$

Define $\varphi_{1}:=\left.\vartheta_{1}\right|_{c_{*}\left(K \times\{0\}, k_{0}\right)}, \psi_{1}:=\left.\vartheta_{1}\right|_{c_{*}\left(K \times\{1\}, k_{0}\right)},\left(\vartheta_{1}\right.$ can be constructed in such a way that $\varphi_{1}, \psi_{1}$ are $k$-chain approximations of $\left.\Phi_{1}, \Phi_{2}\right)$ and define $D \sigma:=\vartheta_{1}(\sigma \times I)$, where $\sigma \times I$ is a chain in $(\tau \times I)^{k_{0}}$.

Now let $\sigma \in \tau^{k_{0}}, \sigma \subset \bar{U}$. Write $\sigma \times I=\sum \sigma_{i}$ (Chain in $(\tau \times I)^{k_{0}}$ ).

Since $\vartheta_{1}$ is a $k$-chain approximation, for any $\sigma_{i}$ there is a point $p\left(\sigma_{i}\right) \in \bar{U} \times I$ such that

$$
\begin{aligned}
\sigma_{i} & \subset s t^{2}\left(p\left(\sigma_{i}\right), k\right) \\
\operatorname{carr} \vartheta_{1} \sigma_{i} & \subset s t\left(F\left(p\left(\sigma_{i}\right)\right), k\right) .
\end{aligned}
$$

$\left(p\left(\sigma_{i}\right)=\left(p_{i}, t_{i}\right), t_{i} \in I.\right)$

Denote by $\bar{\sigma}$ the simplex in $\tau^{l_{0}}$ such that $\sigma \subset \bar{\sigma}$. There is an open set $\mathcal{O}_{x} \in \omega_{1}$ such that $\operatorname{st}^{2}\left(\bar{\sigma}, l_{0}\right) \subset \mathcal{O}_{x}$. Therefore,

$$
F\left(s t^{2}(\sigma, k) \times I\right) \subset \operatorname{st}(F(x \times I), l) .
$$

Because of $p_{i} \in s t^{2}(\sigma, k), \operatorname{carr} \vartheta_{1} \sigma_{i} \subset s t^{2}(F(x \times I), l)$. Thus, carr $D \sigma \subset$ $s t^{2}(F(x \times I), l)$.

Set $d(\sigma):=x$, and the proof is finished.

THEOREM 4.4. The fixed point index ' $i(K, \Phi, U)$ ' for acyclic maps has the following properties:

I. HOMOTOPY INVARIANCE: Let $F: \bar{U} \times I \rightarrow K$ be an acyclic homotopy joining $\left.\Phi_{1}\right|_{\bar{U}}$ and $\left.\Phi_{2}\right|_{\bar{U}}$ such that $\left(K, F_{t}, U\right)$ is admissible, $t \in I$. Then

$$
i\left(K, \Phi_{1}, U\right)=i\left(K, \Phi_{2}, U\right)
$$

II. AdDitivity. Let $U_{1}, U_{2} \subset U$ be open, disjoint subsets of $U$. If $\operatorname{Fix}\left(\left.\Phi\right|_{\bar{U}}\right) \subset U_{1} \cup U_{2}$, then

$$
i(K, \Phi, U)=i\left(K, \Phi, U_{1}\right)+i\left(K, \Phi, U_{2}\right) .
$$

III. Normalization. $i(K, \Phi, K)=\Lambda\left(\Phi_{*}: H_{*}(K) \rightarrow H_{*}(K)\right)$.

Definition 4.5. Let $X_{0}=K, X_{1}, \cdots, X_{n+1}=K$ be compact polyhedra, and let $\Phi_{i}: X_{i} \rightarrow X_{i+1}, i=0, \cdots, n$ be acyclic. Let $U \subset K$ be open, and let $(K, \Phi, U)$ be admissible where $\Phi=\Phi_{n} \circ \cdots \circ \Phi_{0}$.

Then

$$
i(K, \Phi, U):=I_{A}(K, \Phi, U),
$$

where $A=A\left(\Phi_{n}\right) \circ \cdots \circ A\left(\Phi_{0}\right)$ for regular $A$-systems $A\left(\Phi_{i}\right)$ of $\Phi_{i}$, is 
called the fixed point index of $\Phi$ in $U$.

Because of 4.1 the definition is independent of the choice of $A$-systems $A\left(\Phi_{i}\right)$.

Commutativity 4.6. Let $\Phi_{1}: K \rightarrow L, \Phi_{2}: L \rightarrow K$ be acyclic, and let $\left(K, \Phi_{2} \Phi_{1}, U\right)$ and $\left(L, \Phi_{1} \Phi_{2}, \Phi_{2}^{-1}(U)\right)$ be admissible. Assume that for all $y \in \operatorname{Fix}\left(\Phi_{1} \Phi_{2}\right) \backslash \Phi_{2}^{-1}(U)$,

$$
\Phi_{2}(y) \cap \operatorname{Fix}\left(\left.\Phi_{2} \Phi_{1}\right|_{\bar{U}}\right)=\varnothing
$$

Then

$$
i\left(K, \Phi_{2} \Phi_{1}, U\right)=i\left(L, \Phi_{1} \Phi_{2}, \Phi_{2}^{-1}(U)\right)
$$

COROLLARY 4.7 (Reduction property) [15]. Let $U \subset K$ be open, and let $L \subset K$ be a polyhedron such that $\Phi(K) \subset L$. Let $(K, \Phi, U)$ be admissible. Then

$$
i(K, \Phi, U)=i\left(L,\left.\Phi\right|_{L}, L \cap U\right) .
$$

MOD-P PROPERTY 4.8. Let $p \in N$ be prime, and let $(K, \Phi, U)$ and $\left(K, \Phi^{p}, U\right)$ be admissible.

Assume that for all $y \in \operatorname{Fix}\left(\Phi^{p}\right) \backslash U$

$$
\Phi^{k}(y) \cap \operatorname{Fix}\left(\left.\Phi^{p}\right|_{\bar{U}}\right)=\varnothing, \quad 1 \leqq k<p .
$$

Then

$$
i(K, \Phi, U)=i\left(K, \Phi^{p}, U\right) \text { in } Z_{p}
$$

Proofs of 4.4-4.8. The homotopy invariance follows from 4.3, 2.14 and from 1.5. The additivity follows from 2.8. The normalization follows from 3.9 and the Hopf trace formula, e.g., [6, p. 6].

The commutativity follows from 2.17. and the mod-p property follows from 2.19 .

Concluding remarks. (1) The results of the paper were proved for mappings on compact polyhedra. But, it should be possible to extend our fixed point index (including all properties) to compact metric ANR's by using retraction arguments, see [6], or by generalizing the theory to Browder's semi-complexes.*

(2) There are two natural questions which remain unsolved:

Problem I. How is the fixed point index introduced by Calvert

* Added in proof: See G. Skordev, Fixed point index for ANR's preprint, University of Bremen, July 1981. 
[7] related to the fixed point index presented here?

Since regularization techniques (e.g., the 'Hopf construction' [23], [6]) do not apply to acyclic maps, the problem can not be solved in the way known from single-valued maps.

Because of the same reason it is unclear whether fixed point index for acyclic mappings is uniquely determined by certain axioms:

Problem II. Is a fixed point index for acyclic maps (say on polyhedra) uniquely determined by the properties Homotopy invariance, Additivity, Normalization and Reduction property?

APPENDIX. In this appendix we give proofs of Lemma 3.5 and of Lemma 3.8. In order to prove Lemma 3.5 we need two further lemmas.

Lemma A. Let $K$ be a compact polyhedron with triangulation $\tau$, and let $C \subset K$ be a closed subset of $K$ which is acyclic, $\widetilde{H}_{*}(C ; \boldsymbol{F})=0$ $\left(\widetilde{H}_{*}=\right.$ reduced Čech-homology; $\boldsymbol{F}=$ field $)$.

For $l \geqq k$ let $i_{l, k}: C_{l} \subseteq C_{k}$ be the inclusion, where

$$
C_{j}:=s t(C, j), \quad j \in N \text {. }
$$

Then for all $k \in N$ there is $k_{0} \in N$ such that

$$
\left(i_{k_{0}, k}\right)_{*}: \widetilde{H}_{*}\left(C_{k_{0}}\right) \longrightarrow \widetilde{H}_{*}\left(C_{k}\right)
$$

is trivial.

Proof. The proof follows from the continuity of Čech-homology, see [13].

Lemma B. Let $(K, \tau),(L, \mu)$ be compact polyhedra with triangulations $\tau$ and $\mu$, and let $\Phi: K \rightarrow L$ be acyclic. Let $k_{0}, n \in N$.

There are numbers $k_{1}, \cdots, k_{n+1} \in N$ such that for any simplex $\sigma \in \tau^{k_{i}}$ there exists an integer $k_{i}(\sigma), 0 \leqq i \leqq n$, and there exists $a$ point $a(\sigma) \in K$ such that

(1) $k_{0}<k_{1}<\cdots<k_{n+1}$

$$
k_{i}>k_{i-1}(\sigma) \text { for all } \sigma \in \tau^{k_{2}} \text {. }
$$

(2) For any simplex $\sigma \in \tau^{k_{i}}$

$$
\begin{aligned}
& \sigma \subset s t^{2}\left(a(\sigma), k_{i-1}\right) \\
& \Phi\left(s t^{3}\left(\tau, k_{i}\right)\right) \subset s t\left(\Phi(a(\sigma)), k_{i-1}(\sigma)\right)
\end{aligned}
$$


(3) $i_{*}: \tilde{H}_{*}\left(s t^{2}\left(\Phi(a(\sigma)), k_{i-1}(\sigma)\right) \rightarrow \tilde{H}_{*}\left(s t\left(\Phi(a(\sigma)), k_{i-1}\right)\right)\right.$ is trivial $(i=$ inclusion $)$.

Proof. The proof proceeds by induction on $n$. We give a proof only for $n=0$.

Because of Lemma $\mathrm{A}$ for any $y \in K$ there is $k_{0}(y) \in N$ such that

$$
i_{*}: \widetilde{H}_{*}\left(s t^{2}(\Phi(y)), k_{0}(y) \longrightarrow \widetilde{H}_{*}\left(s t\left(\Phi(y), k_{0}\right)\right)\right.
$$

is trivial ( $i=$ inclusion).

Moreover, for $y \in K$ there is a neighborhood $\mathcal{O}_{y} \subset K$ of $y$ such that $\Phi\left(\mathscr{O}_{y}\right) \subset \operatorname{st}\left(\Phi(y), k_{0}(y)\right)$ and such that $\mathscr{O}_{y}$ is contained in some element of $s t\left(\tau^{k_{0}}\right)$. Let $\omega_{1}=\left\{\mathcal{O}_{y_{i}}\right\}_{i=1}^{s}$ be a finite subcovering of $\omega=$ $\left\{\mathcal{O}_{y}\right\}_{y \in K}$. Choose $k_{1} \in N$ such that $k_{1}>\max \left\{k_{0}, k_{0}\left(y_{1}\right), \cdots, k_{0}\left(y_{s}\right)\right\}$ and such that

$$
\left\{\text { int } s t^{3}\left(\sigma, k_{1}\right) \mid \sigma \in \tau^{k_{1}}\right\}
$$

is a refinement of $\omega_{1}$.

For $\sigma \in \tau^{k_{1}}$ define $a(\sigma):=y_{i}$, where $s t^{3}\left(\sigma, k_{1}\right) \subset \mathcal{O}_{y_{i}}$, and $k_{0}(\sigma):=$ $k_{0}\left(y_{i}\right)$.

Proof of Lemma 3.5. Let $l \in N$, and let $\Phi: K \rightarrow L$ be acyclic.

Applying Lemma B with $k_{0}=l$ and $n=\operatorname{dim} K$ we obtain a sequence of numbers $k_{0}<k_{1}<\cdots<k_{n+1}$.

Define $l_{0}:=k_{n+1}$.

We will construct successively a chain map

$$
\tilde{\varphi}_{1}: C_{*}\left(K, l_{0}\right) \longrightarrow C_{*}\left(L, l_{0}\right)
$$

with the following properties:

(1) $\widetilde{\varphi}_{1}$ is augmentation preserving

(2) For any simplex $\sigma \in \tau^{l_{0}}$ with $\operatorname{dim} \sigma=i$ there is a point $p(\sigma) \in K$ such that

$$
\begin{aligned}
\sigma & \subset s t^{2}\left(p(\sigma), k_{n-i}\right) \\
\operatorname{carr} \widetilde{\varphi}_{1} \sigma & \subset \operatorname{st}\left(\Phi(p(\sigma)), k_{n-i}\right) .
\end{aligned}
$$

Composing $\widetilde{\varphi}_{1}$ with $\chi: C_{*}\left(L, l_{0}\right) \rightarrow C_{*}(L, l)$ we obtain the desired chain approximation of $\Phi$ :

$$
\varphi_{1}:=\chi \widetilde{\varphi}_{1}
$$

Construction of $\widetilde{\varphi}_{1}$ :

$i=0$ : Let $\sigma_{0}$ be a vertex of $\tau^{l_{0}}$. Because of Lemma B there is a point $a\left(\sigma_{0}\right) \in K$. Set $\widetilde{\varphi}_{1}\left(\sigma_{0}\right)=a$, where $a$ is a vertex of $\mu^{l_{0}}$ which is contained in $\operatorname{st}\left(\Phi\left(a\left(\sigma_{0}\right)\right), k_{n}\right)$. Define $p\left(\sigma_{0}\right):=a\left(\sigma_{0}\right)$. 
$i>0$ : Assume $\widetilde{\varphi}_{1}$ is defined on simplices $\sigma$ of dimension $i-1$, and satisfies the conditions (1) and (2).

Let $\sigma \in \tau^{l_{0}}$ with $\operatorname{dim} \sigma=i$.

Let $\bar{\sigma} \in \tau^{k_{n+1-i}}$ such that $\operatorname{dim} \bar{\sigma}=i$ and $\bar{\sigma} \supset \chi(\sigma)$.

Because of Lemma B there is a point $a(\bar{\sigma})$ and a number $k_{n-i}(\bar{\sigma})$. Write $\partial \sigma=\sum \sigma_{s}\left(\operatorname{dim} \sigma_{s}=i-1\right)$.

By assumption there are points $p\left(\sigma_{s}\right)$ such that

$$
\begin{aligned}
\sigma_{s} & \subset s t^{2}\left(p\left(\sigma_{s}\right), k_{n+1-i}\right) \\
\operatorname{carr} \widetilde{\varphi}_{1} \sigma_{s} & \subset s t\left(\Phi\left(p\left(\sigma_{s}\right)\right), k_{n+1-i}\right) .
\end{aligned}
$$

Since $\Phi\left(p\left(\sigma_{s}\right)\right) \subset s t\left(\Phi(a(\bar{\sigma})), k_{n-i}(\bar{\sigma})\right)$

$$
\operatorname{carr} \widetilde{\varphi}_{1} \partial \sigma \subset s t^{2}\left(\Phi(a(\bar{\sigma})), k_{n-i}(\bar{\sigma})\right) .
$$

By our inductive assumption, the following is a cycle

$$
\widetilde{\varphi}_{1} \partial \sigma \in C_{i-1}\left(s t^{2}\left(\Phi(a(\bar{\sigma})), k_{n-i}(\bar{\sigma})\right), l_{0}\right) \text {. }
$$

Hence, by Lemma B there is a chain $c \in C_{i}\left(\operatorname{st}\left(\Phi(a(\bar{\sigma})), k_{n-i}\right), l_{0}\right)$ such that $\partial c=\tilde{\varphi}_{1} \partial \sigma$.

Thus, define $\widetilde{\varphi}_{1}(\sigma):=c, p(\sigma):=a(\bar{\sigma})$.

Proof of Lemma 3.8. Let $j \in N$, and let $\Phi: K \rightarrow L$ be acyclic. Applying Lemma B with $k_{0}=j$ and $n=\operatorname{dim} K$ we obtain a sequence of numbers $k_{0}<k_{1}<\cdots<k_{n+1}$.

Define $j_{1}:=k_{n+1}$.

Let $m \geqq l \geqq j_{1}$, and let $\varphi=\varphi_{1} b \in A^{*}(\Phi)_{l}, \psi=\psi_{1} b \in A^{*}(\Phi)_{m}$,

$$
\begin{aligned}
& \varphi_{1}: C_{*}\left(K, l_{1}\right) \longrightarrow C_{*}(L, l) \\
& \psi_{1}: C_{*}\left(K, m_{1}\right) \longrightarrow C_{*}(L, m) .
\end{aligned}
$$

We will construct successively a homotopy $D$,

$$
D_{i}: C_{i}\left(K, m_{1}\right) \longrightarrow C_{i+1}(L, l)
$$

such that

(1) $\chi_{\psi_{1}}-\varphi_{1} \chi=\partial D_{i}+D_{i-1} \partial$

(2) For any simplex $\sigma \in \tau^{m_{1}}, \operatorname{dim}=i$, there is a point $c(\sigma) \in K$ such that

$$
\begin{aligned}
\sigma & \subset s t^{3}\left(c(\sigma), k_{n-i}\right) \\
\operatorname{carr} D_{i} \sigma & \subset s t\left(\Phi(c(\sigma)), k_{n-i}\right) .
\end{aligned}
$$

Construction of $D$ :

$i=0$ : Let $\sigma_{0}$ be a vertex of $\tau^{m_{1}}$, and set $\bar{\sigma}:=\chi\left(\sigma_{0}\right) \in \tau^{l_{1}}, \tilde{\sigma}:=$ $\chi\left(\sigma_{0}\right) \in \tau^{j_{1}}$. Since $\varphi_{1}$ is a chain approximation we find a point $p(\bar{\sigma})$ satisfying the conditions of 3.5 , and since $\psi_{1}$ is a chain approximation 
we find a point $p\left(\sigma_{0}\right)$ satisfying the conditions of 3.5. Moreover, from Lemma B we find a point $a(\tilde{\sigma}) . \quad p(\bar{\sigma}), p\left(\sigma_{0}\right)$ and $a(\tilde{\sigma})$ have the following properties:

$$
\begin{aligned}
& p(\bar{\sigma}), p\left(\sigma_{0}\right) \subset s t^{3}\left(\widetilde{\sigma}, j_{1}\right) \\
& \Phi(p(\bar{\sigma})) \cup \Phi\left(p\left(\sigma_{0}\right)\right) \subset \operatorname{st}\left(\Phi(a(\widetilde{\sigma})), k_{n}(\widetilde{\sigma})\right) \\
& \operatorname{carr} \chi_{\psi_{1} \sigma_{0}} \cup \operatorname{carr} \varphi_{1} \chi_{\sigma_{0}} \subset s t^{2}\left(\Phi(a(\widetilde{\sigma})), k_{n}(\widetilde{\sigma})\right) .
\end{aligned}
$$

Because of Lemma B,

$$
i_{*}: \widetilde{H}_{*}\left(s t^{2}\left(\Phi(a(\widetilde{\sigma})), k_{n}(\widetilde{\sigma})\right)\right) \longrightarrow \widetilde{H}_{*}\left(s t\left(\Phi(a(\widetilde{\sigma})), k_{n}\right)\right)
$$

is trivial ( $i=$ inclusion).

Since $\varphi_{1}, \psi_{1}$ are augmentation preserving, and since $l>k_{n}$, there is a chain $c_{1} \in C_{1}\left(s t\left(\Phi(\alpha(\widetilde{\sigma})), k_{n}\right), l\right)$ such that

$$
\partial c_{1}=\chi_{\psi_{1} \sigma_{0}}-\varphi_{1} \chi \sigma_{0} .
$$

Set $D_{0} \sigma_{0}:=c_{1}, c\left(\sigma_{0}\right):=a(\tilde{\sigma})$.

$i>0:$ Assume $D_{j}$ is defined for $j<i$ and satisfies the desired properties.

Let $\sigma \in \tau^{m_{1}}, \operatorname{dim} \sigma=i$.

Let $\bar{\sigma} \in \tau^{l_{1}}$ be the simplex with $\chi(\sigma) \subset \bar{\sigma}$, dim $\bar{\sigma}=i$, let $\tilde{\sigma} \in \tau^{k_{n+1-i}}$ be the simplex with $\chi(\sigma) \subset \widetilde{\sigma}, \operatorname{dim} \widetilde{\sigma}=i$.

Since $\varphi_{1}$ is a chain approximation we find a point $p(\bar{\sigma})$, and since $\psi_{1}$ is a chain approximation we find a point $p(\sigma)$. Moreover, from Lemma $\mathrm{B}$ we find a point $a(\tilde{\sigma}) . \quad p(\bar{\sigma}), p(\sigma)$, and $a(\tilde{\sigma})$ satisfy the following properties:

$$
\begin{aligned}
& p(\sigma), p(\bar{\sigma}) \subset s t^{3}\left(\tilde{\sigma}, k_{n+1-i}\right) \\
& \Phi(p(\sigma)) \cup \Phi(p(\bar{\sigma})) \subset s t\left(\Phi(a(\widetilde{\sigma})), k_{n-i}(\widetilde{\sigma})\right) \\
& \operatorname{carr} \chi_{\psi_{1} \sigma} \cup \operatorname{carr} \varphi_{1} \chi_{\sigma} \subset s t^{2}\left(\Phi(a(\widetilde{\sigma})), k_{n-i}(\tilde{\sigma})\right) .
\end{aligned}
$$

Write $\partial \sigma=\sum \sigma_{s}, \operatorname{dim} \sigma_{s}=i-1$.

By assumption there is a point $c\left(\sigma_{s}\right)$ such that

$$
c\left(\sigma_{s}\right) \subset s t^{3}\left(\widetilde{\sigma}, k_{n+1-i}\right) .
$$

Therefore,

$$
\begin{aligned}
\Phi\left(c\left(\sigma_{s}\right)\right) & \subset s t\left(\Phi(a(\widetilde{\sigma})), k_{n-i}(\widetilde{\sigma})\right) \\
\operatorname{carr} D_{i-1} \sigma_{s} & \subset s t^{2}\left(\Phi(a(\widetilde{\sigma})), k_{n-i}(\widetilde{\sigma})\right)
\end{aligned}
$$

Thus,

$$
\operatorname{carr} D_{i-1} \partial \sigma \subset s t^{2}\left(\Phi(a(\widetilde{\sigma})), k_{n-i}(\widetilde{\sigma})\right)
$$


Set $c:=\chi_{\psi_{1}} \sigma-\varphi_{1} \chi_{\sigma}-D_{i-1} \partial \sigma$.

Then $\operatorname{carr}(c) \subset s t^{2}\left(\Phi(a(\tilde{\sigma})), k_{n-i}(\tilde{\sigma})\right)$ and $\partial c=0$.

Because of Lemma $B$ there is a chain

with

$$
c_{i+1} \in C_{i+1}\left(s t\left(\Phi(a(\widetilde{\sigma})), k_{n-i}\right), l\right)
$$

$$
\partial c_{i+1}=c .
$$

Set $D_{i} \sigma:=c_{i+1}, c(\sigma):=a(\widetilde{\sigma})$.

Note added in proof. This single-valued case was treated independently (using essentially O'Neills definition) by G. Fournier in [46].

\section{REFERENCES}

1. E. Begle, The Vietoris mapping theorem for bicompact spaces, Ann. Math., 51 (1950), 534-543.

2. Yu. G. Borisovič, E. D. Gel'man, A. D. Myškis and V. V. Obuhovskiǔ, Topological methods in fixed point theory for multivalued mappings, Uspekhi Mat. Nauk, 35 (1980), 59-126 (in Russian).

3. Yu. G. Borisovič, E. D. Gel'man, E. Muhamadiev, and V. V. Obuhovskiǐ, On the rotation of multivalued vector fields, Dokl. Akad. Nauk SSSR, 187 (1969), 956-959.

4. D. G. Bourgin, Modern Algebraic Topology, The Macmillan Comp., N. Y., 1963.

5. F. E. Browder, On the fixed point index for continuous mappings of locally connected spaces, Summa Brasil. Math., 4 (1960), 253-293.

6. R. F. Brown, The Lefschetz Fixed Point Theorem, Scott Foresman and Co. Glenview, Illinois-London, 1971.

7. B. Calvert, The local fixed point index for multivalued transformations in a Banach Space, Math. Ann., 190 (1970), 119-128.

8. A. Cellina, and A. Lasota, A new approach to the definition of topological degree for multivalued mappings, Lincei Rend. Sci. Mat. e Nat., 47 (1969), 434-440.

9. A. Dold, Fixed point index and fixed point theorem for Euclidean neighborhood retracts, Topology, 4 (1965), 1-8.

10. L Lectures on Algebraic Topology, Springer, Berlin-Heldelberg-New York, 1972.

11. A coincidence fixed point theorem, L'Enseignement Math., 26 (1978), 75-87.

12. S. Eilenberg and D. Montgomery, Fixed point theorems for multi-valued transformations, Amer. J. Math., 58 (1946), 214-222.

13. S. Eilenberg and N. Steenrod, Foundations of Algebraic Topology, Princeton Univ. Press, 1952.

14. E. Fadell, Recent results in the fixed point theory of continuous maps, Bull. AMS,

76 (1970), 10-29.

15. C. Fenske and H. O. Peitgen, Repulsive fixed points of multivalued transformations and the fixed point index, Math. Ann., 218 (1975), 9-18.

16. R. Geoghegan, The homomorphism on fundamental group induced by a homotopy idempotent having essential fixed points, Inst. for Adv. Study, Princeton, preprint.

17. L. Górniewicz, Homological methods in fixed point theory of multivalued maps, Diss. Math., (Rozpr. Mat.), 129 (1976), 71 pp.

18. — A review of various results and problems of the fixed point theory of multivalued mappings, Univ. Gdansk, Inst. Math., preprint 1, 1978. 
19. A. Granas, Sur la notion du degree topologique pour une certaine classe de transformations multivalentes dans espaces de Banach, Bull. Acad. Polon. Sci., 7 (1959), $271-275$

20. A. Granas and J. W. Jaworowski, Some theorems on multivalued mappings of subsets of the Euclidean space, Bull. Acad. Polon. Sci. Ser. math. astr. et phys., 7 (1959), 277-283.

21. B. A. Halpern, A general coincidence theory, Pacific J. Math., 77 (1978), 451-471.

22. P. J. Hilton and S. Wylie, Homology Theory, Cambr. Univ. Press, 1960.

23. H. Hopf, Über die algebraische Anzahl von Fixpunkten, Math. Z., 29 (1929), 429-462.

24. S. Kakutani, A generalization of Brouwer's fixed point theorem, Duke Math. J., 8 (1941), 457-459.

25. M. Krasnosel'skiǐ and P. Zabreǐko, Geometric methods on nonlinear analysis, Nauka Moskow, 1975 (in Russian).

26. Z. Kucharski, A coincidence index, Bull. Acad. Polon. Sci., 4 (1976), 245-252.

27. S. Lefschetz, Intersections and transformations of complexes and manifolds, Trans. AMS, 28 (1926), 1-49.

28. J. Jeray, Sur la position d'un ensemble ferme de points d'un espace topologique. Sur les equations et les transformations, J. de Math. Pures et Appl., 24 (1945), 169-248. 29. - Fixed point index and Lefschetz number, in: Sympos. on infinite dimens. topology, Princeton Univ. Press, 1972.

30. J. Leray and J. Schauder, Topologie et equations functionelles, Ann. Sci. Ec. Norm. Sup., 51 (1934), 221-233.

31. A. Mas-Colell, $A$ note on a theorem of $F$. Browder, Mathematical Programming, 6 (1974), 229-233.

32. A. D. Myškis, Generalization of the theorem on a fixed point of a dynamical system inside of a closed trajectory, Mat. Sborn., 34 (1954), 525-540 (in Russian).

33. J. von Neumann, Über ein ökonomisches Gleichungssystem und eine Verallgemeinerung des Brouwerschen Fixpunktsatzes, Ergebn. math. Kolloqu., 8 (1937), 73-83.

34. B. O'Neill, Essential sets and fixed points, Amer. J. Math., 75 (1953), 497-509.

35. - Induced homology homomorphism for set maps, Pacific J. Math., 7 (1957), 1179-1184.

36. H. O. Peitgen, On the Lefschetz number for iterates of continuous mappings, Proc. AMS, 54 (1976), 441-444.

37. H. O. Peitgen, Methoden der topologischen Fixpunkttheorie in der nichtlinearen Funktionalanalysis, Habilitationsschrift, Univ. Bonn, 1976.

38. M. Powers, Lefschetz fixed point theorem for a new class of multivalued maps, Pacific J. Math., 42 (1972), 211-220.

39. E. Spanier, Algebraic Topology, McGraw-Hill, N. Y., 1966.

40. H. Steinlein, Ein Satz über den Leray-Schauderschen Abbildungsgrad, Math. Z., 126 (1972), 176-208.

41. - A new proof of the $(\bmod p)$-theorem in asymptotic fixed point theory, Proc. Conf. on Problems in Nonlin. Funct. Anal. Bonn, 1974, ed.: Ber. Ges. Math. Datenv. Bonn, 103 (1975), 29-42.

42. A. J. Tromba, The beer barrel theorem, Springer Lecture Notes in Math., vol. 730, p. $485-488$.

43. - A general asymptotic fixed point theorem, Univ. Bonn, SFB 72, preprint no. 411,1980 .

44. L. Vietoris, Über den höheren Zusammenhang kompakter Räume und eine Klasse von zusammenhangstreuen Abbildungen, Math. Ann., 97 (1927), 454-472.

45. P. Zabrěko and M. Krasnosel'kiî, Iterations of operators and fixed points, Dokl. Akad. Nauk SSSR, 196 (1971), 1006-1009.

46. G. Fournier, A Simplicial Approach to the Fixed Point Index, In 'Fixed Point Theory' Proc. Conf. Sherbrooke, Can. 1980, Springer Lecture Notes, vol. 886 (1981), 73-102. 
Received March 30, 1981 and in revised form August 10, 1981. This paper was written while the second author was a research fellow sponsored by the Alexander von Humboldt foundation at the University of Bremen.

UNIVERSITY OF BREMEN, Fachbereich Mathematik

ForschungsschWERPUNKT 'Dynamische Systeme'

(DFG-Projekt "Multiple Bifurkation”)

WEST GERMANY

Center of Mathematics and Mechanics,

1090 SOFIA,

P.O. 373, BulgaRIA 


\section{PACIFIC JOURNAL OF MATHEMATICS}

\section{EDITORS}

DONALD BABBITT (Managing Editor)

University of California

Los Angeles, CA 90024

Hugo RossI

University of Utah

Salt Lake City, UT 84112

C. C. MOORE and ARThur Agus

University of California

Berkeley, CA 94720
J. DugundJI

Department of Mathematics

University of Southern California

Los Angeles, CA 90007

R. FINN and J. MILGRAM

Stanford University

Stanford, CA 94305

\section{ASSOCIATE EDITORS}
R. ARENS
E. F. BeCKENBACH
B. H. NeumanN
F. WOLF
K. YoSHIDA

\section{SUPPORTING INSTITUTIONS}

UNIVERSITY OF ARIZONA

UNIVERSITY OF BRITISH COLUMBIA

CALIFORNIA INSTITUTE OF TECHNOLOGY

UNIVERSITY OF CALIFORNIA

MONTANA STATE UNIVERSITY

UNIVERSITY OF NEVADA, RENO

NEW MEXICO STATE UNIVERSITY

OREGON STATE UNIVERSITY

\author{
UNIVERSITY OF OREGON \\ UNIVERSITY OF SOUTHERN CALIFORNIA \\ STANFORD UNIVERSITY \\ UNIVERSITY OF AAWAII \\ UNIVERSITY OF TOKYO \\ UNIVERSITY OF UTAH \\ WASHINGTON STATE UNIVERSITY \\ UNIVERSITY OF WASHINGTON
}

The Supporting Institutions listed above contribute to the cost of publication of this Journal, but they are not owners or publishers and have no responsibility for its content or policies,

Mathematical parers intended for publication in the Pacific Journal of Mathematics should be in typed form or offset-reproduced, (not dittoed), double spaced with large margins. Please do not use built up fractions in the text of the manuscript. However, you may use them in the displayed equations. Underline Greek letters in red, German in green, and script in blue. The first paragraph or two must be capable of being used separately as a synopsis of the entire paper. Please propose a heading for the odd unmbered pages of less than 35 characters. Manuscripts, in triplicate, may be sent to any one of the editors. Please classify according to the scheme of Math. Reviews, Index to Vol. 39. Supply name and address of author to whom proofs should be sent. All other communications should be addressed to the managing editor, or Elaine Barth, University of California, Los Angeles, California, 90024.

50 reprints to each author are provided free for each article, only if page charges have been substantially paid. Additional copies may be obtained at cost in multiples of 50 .

The Pacific Journal of Mathematics is issued monthly as of January 1966, Regular subscription rate: $\$ 114.00$ a year (6 Vol., 12 issues). Special rate: $\$ 57.00$ a year to individual members of supporting institution.

Subscriptions, orders for numbers issued in the last three calendar years, and changes of address shoud be sent to Pacific Journal of Mathematics, P.O. Box 969, Carmel Valley, CA 93924, U.S.A. Old back numbers obtainable from Kraus Periodicals Co., Route 100, Millwood, NY 10546.

\section{PUBLISHED BY PACIFIC JOURNAL OF MATHEMATICS, A NON-PROFIT CORPORATION}

Printed at Kokusai Bunken Insatsusha (International Academic Printing Co., Ltd.). 8-8, 3-chome, Takadanobaba, Shinjuku-ku, Tokyo 160, Japan. 


\section{Pacific Journal of Mathematics}

\section{Vol. 102, No. 2 \\ February, 1982}

Richard A. Boyce, Irreducible representations of finite groups of Lie type through block theory and special conjugacy classes ...............253

Robert Jay Daverman and Dennis J. Garity, Intrinsically

$(n-2)$-dimensional cellular decompositions of $E^{n} \ldots \ldots \ldots \ldots 275$

Juan Ferrera, Spaces of weakly continuous functions ................285

William George Frederick, $\mu$-theta functions ................... 293

Christopher George Gibson and T. D. Ward, On stratifying pairs of linear

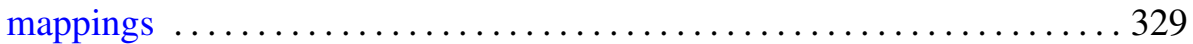

Stanley Joseph Gurak, Minimal polynomials for Gauss circulants and cyclotomic units ........................................ 347

Joachim Georg Hartung, On two-stage minimax problems ............. 355

Robert P. Kaufman, Hausdorff measure, BMO, and analytic functions . . . . 369

Neal I. Koblitz, $p$-adic analog of Heine's hypergeometric $q$-series . . . . . . . 373

Kurt Kreith, Picone-type theorems for hyperbolic partial differential

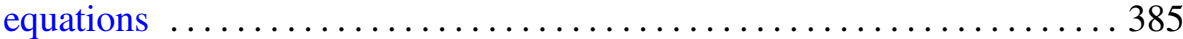

Nicholas J. Kuhn, The geometry of the James-Hopf maps ............. 397

Donald Michael Redmond, Explicit formulae for a class of Dirichlet

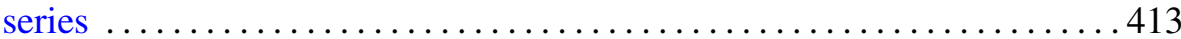

J. R. Respess and Elliott Ward Cheney, Jr., Best approximation problems in tensor-product spaces . .............................. 437

Allen Ross Schweinsberg, The operator equation $A X-X B=C$ with normal $A$ and $B$

Hans-Willi Siegberg and Guentcho Svetoslavov Skordev, Fixed point index and chain approximations

Kondagunta Sundaresan, Geometry and nonlinear analysis in Banach spaces 